\title{
Blocking preferential glucose uptake sensitizes liver tumor-initiating cells to glucose restriction and sorafenib \\ treatment
}

Hui-Lu Zhang ${ }^{1, \dagger}$, Ming-Da Wang ${ }^{2, \dagger}$, Xu Zhou ${ }^{2, \dagger}$, Chen-Jie Qin ${ }^{2, \uparrow}$, Gong-Bo Fu ${ }^{2}$, Liang Tang ${ }^{2,3}$, Han Wu ${ }^{2}$, Shuai Huang ${ }^{2}$, Ling-Hao Zhao ${ }^{2}$, Min Zeng ${ }^{2}$, Jiao Liu $^{2}$, Dan $\mathrm{Cao}^{2}$, Lin-Na Guo ${ }^{2}$, Hong-Yang Wang, 2,3," He-Xin $\operatorname{Yan}^{2,3, *}, \mathrm{Jie} \mathrm{Liu}^{1, *}$.

\section{Authors address:}

${ }^{1}$ Department of Digestive Diseases, Huashan Hospital, Fudan University, Shanghai 200040, China;

${ }^{2}$ International Cooperation Laboratory on Signal Transduction, Eastern Hepatobiliary Surgery Institute, the Second Military Medical University, Shanghai 200438, China;

${ }^{3}$ National Center for Liver Cancer Research, Shanghai 201805, China;

${ }^{\dagger}$ These authors have contributed equally to this work.

*Corresponding authors. Address correspondence to: International Cooperation Laboratory on Signal Transduction, Eastern Hepatobiliary Surgery Institute, the Second Military Medical University, 225 Changhai Road, Shanghai 200438,Tel: +86 218187 5361; fax: +86 21 65566851. E-mail: hywangk@vip.sina.com (H.-Y. Wang) and hexinyw@163.com (H.-X. Yan); Department of Digestive Diseases, Huashan Hospital, Fudan University, 12 Wulumuqi Road, Shanghai 200040, Tel: +86 21 52888236. E-mail: jieliu@ fudan.edu.cn (J.L). 


\section{Abstract}

Cancer cells display altered metabolic phenotypes characterized by a high level of glycolysis, even under normoxic conditions. Because of a high rate of glycolytic flux and inadequate vascularization, tumor cells often suffer from nutrient deficiency and require metabolic adaptations to address such stresses. Although tumor-initiating cells (T-ICs) have been identified in various malignancies, the cells' metabolic phenotypes remain elusive. In this study, we observed that liver T-ICs preferentially survived under restricted glucose treatment. These cell populations compete successfully for glucose uptake by preferentially expressing glucose transporters (GLUT1 and GLUT3), whereas inhibition of GLUT1 or GLUT3 abolished the survival advantage and suppressed the tumorigenic potential of liver T-ICs. Among signaling pathways related to T-ICs, IL-6/STAT3 was identified to be responsible for the elevation of glucose uptake in liver T-ICs under glucose limitation. Further investigation revealed that IL-6 stimulation upregulated GLUT1 and GLUT3 expressions in $\mathrm{CD}_{133^{+}}$cells, particularly during glucose deprivation. More importantly, inhibition of glucose uptake sensitized liver T-ICs to sorafenib treatment and enhanced the therapeutic efficacy in vivo. Our findings suggest that blocking IL-6/STAT3-mediated preferential glucose uptake might be exploited for novel therapeutic targets during hepatocellular carcinoma (HCC) progression.

Keywords: tumor-initiating cells, nutrition stress, glucose uptake, GLUT1/3, IL-6/STAT3, sorafenib 
Abbreviations: Human hepatocellular carcinoma (HCC); tumor initiating cells (T-ICs); $\left[{ }^{18} \mathrm{~F}\right]$ deoxyglucose positron emission tomography $\left({ }^{18}\right.$ FDG-PET); Phloretin (Phl); 2-[N-(7-nitrobenz-2-oxa-1,3-diaxol-4-yl)amino]-2-deoxyglucose (2-NBDG); 2-deoxy-D-glucose (2DG); small interfering RNAs (siRNA); small hairpin RNAs (shRNA) .

\section{Conflict of interest statement:}

The authors who have taken part in this study declared that they do not have anything to disclose regarding funding or conflict of interest with respect to this manuscript.

\section{Financial support:}

This work was supported by the projects from State key infection disease project of China (2012ZX10002), National Science Foundation of China (31371440), Shanghai Outstanding Academic Scholarship (16XD1403300). 


\section{Introduction}

Human hepatocellular carcinoma (HCC) is among the top five most deadly cancers worldwide and is particularly prevalent in China [1, 2]. Although surgical resection is a potentially curative therapy for HCC patients, this disease is still difficult to treat because of high rates of recurrence and chemotherapy resistance $[3,4]$. Increasing evidence suggests that liver cancer stem cells (or tumor-initiating cells, T-ICs) can give rise to tumor heterogeneity and are particularly critical to hepatocarcinogenesis [5-7]. The T-ICs are a small subset of cancer cells bearing stem cell-like features and strong self-renewal capacities, which are indispensable to initiate and maintain tumor growth [8]. It is widely accepted that a variety of stem cell biomarkers including CD133 [9], EpCAM [7], CD90 [6], CD24 [10] and OV6 [11] could be used for identification of liver T-ICs. Moreover, the isolated biomarker-positive T-ICs are more metastatic and resistant to conventional chemotherapies, which may be responsible for cancer relapse [12]. Thus, liver T-ICs might be an attractive target for HCC treatment.

Unlike normal cells, cancer cells largely rely on glycolysis for ATP generation rather than mitochondrial oxidative phosphorylation even under normal oxygen concentrations [13],[14]. This peculiar tumor-specific metabolic profile is termed "Warburg effect" and has been documented in different human tumor types[15],[16]. It is also exploited clinically for tumor detection with $\left[{ }^{18} \mathrm{~F}\right]$ de-oxyglucose positron emission tomography (FDG-PET) imaging [17]. Although T-ICs have been identified in various malignant tumors [18], the metabolic profile of the T-IC subset has not yet been elucidated.

Because of the high rate of glycolytic flux and lack of vasculature, nutrient concentration in solid tumors, particularly glucose, is generally lower than that in normal tissues [19],[20]. 
Thus, cancer cells must adapt their metabolic phenotypes to the unfavorable microenvironment. It has been reported that hypoxic stimulation facilitates the formation and expansion of glioma stem cells [21], whereas disrupting hypoxia-inducible factor (HIF) eliminated the fractions of cancer stem-like cells in hematological malignancies [22]. Glucose restriction could also enrich brain T-ICs and promote tumor progression by enhancing survival pathways [23]. All these findings highlight the importance of understanding the mechanisms driving metabolic alternations in cancer, particularly within the T-ICs subset, for development of targeted therapies. However, whether the metabolic stress could affect functions of liver T-ICs during HCC progression is still unclear.

In the present study, we demonstrated that glucose restriction promoted the enrichment of liver T-ICs, which also preferentially took up glucose and survived under low-glucose conditions. We also showed that activation of the IL-6/STAT3 signaling cascade was involved in the regulation of glucose transporters-mediated glucose uptake in liver T-ICs. More importantly, inhibition of glucose uptake significantly suppressed T-ICs-initiated tumor formation and sensitized T-ICs to sorafenib treatment in vivo. These data suggested that Gluts-mediated glucose uptake was pivotal for liver T-ICs to adapt to nutritional deficienciesand can be exploited to identify potential therapeutic targets against HCC.

\section{Materials and Methods}

\subsection{Clinical HCC tissues}

This study was approved by the Eastern Hepatobiliary Surgery Hospital Research Ethics Committee. Fresh human liver cancer specimens were obtained from patients who underwent 
curative hepatectomy for HCC at Eastern Hepatobiliary Surgery Hospital. Primary HCC cells were isolated from fresh HCC specimens as previously described [24]. Another 87 HCC samples were collected for qRT-PCR assay (Supplementary Table 1). All patients provided written informed consent.

Additional methods are available in the supplementary information.

\section{Results}

\subsection{Enriched liver T-ICs preferentially survived under restricted glucose conditions.}

To test if glucose restriction could impact the liver T-IC subpopulation, we incubated human HCC cell lines (Huh7 and MHCC-97L) in cultural media containing standard (25mM) or limited (2.5mM) levels of glucose. Because of the high rate of glucose consumption by tumor cells and a limited blood supply, glucose concentrations in solid tumors are generally three- to ten-fold lower than that in normal tissues [19],[25]. In the present study, the condition of "restricted" glucose referred to the media containing one-tenth of the normal glucose concentration. The flow cytometric assay revealed a two-fold or greater increase in the proportion of HCC cells expressing the putative T-IC surface marker CD133 in the cultures with limited glucose (Fig. 1A). Similarly, elevated percentages of the EpCAM-positive subset were also observed in Huh7 and MHCC-97L cells after culture in low glucose (Fig. 1B). We next replaced glucose with the non-metabolized glucose analog 2-deoxy-D-glucose (2DG) [26],[27] to mimic the restricted-glucose condition. As shown in Fig. 1C, 2DG treatment enriched the fraction of $\mathrm{CD} 133^{+} \mathrm{T}-\mathrm{ICs}$ in a concentration-dependent manner. To further confirm this regulatory effect of glucose deprivation on the liver T-IC subset, we exposed 
primary liver cancer cells isolated from the freshly resected HCC tissues to the standard cultural medium or glucose-restricted medium as described above. Consistent with the results in HCC cell lines, both low-glucose culture and 2DG treatment notably facilitated the enrichment of the $\mathrm{CD}_{133^{+}}$and $\mathrm{EpCAM}^{+}$subsets in the total tumor cell population relative to that of the normal-glucose culture (Fig. 1D). Taken together, these data indicate that glucose deprivation increased the percentage of T-IC subpopulations.

\subsection{Liver T-ICs exhibited elevated glucose uptake capacity.}

To understand the percentage change in the fractions of liver T-ICs associated with glucose limitation, we examined the survival rates of both T-ICs and their matched counterparts under normal and restricted glucose. Using flow cytometry, we found that CD133 ${ }^{+} \mathrm{T}-\mathrm{ICs}$ were resistant to restricted glucose-induced cell apoptosis (Fig. 2A and 2B). In contrast, their matched CD133- counterparts exhibited five times more apoptosis cells than CD133 ${ }^{+} \mathrm{T}-\mathrm{ICs}$ under glucose deprivation, implying that the T-IC subset was resistant to low-glucose conditions. As glucose is the major carbon source for the maintenance of tumor cells, efficient glucose uptake may confer cancer cells a survival advantage under conditions with limited glucose. Thus, it is plausible to speculate that the liver T-ICs may possess higher glucose uptake capacity than their corresponding non-TIC subpopulations. To test this hypothesis, we incubated HCC cells with a fluorescent D-glucose analog 2-[N-(7-nitrobenz-2-oxa-1,3-diaxol-4-yl)amino]-2-deoxyglucose (2-NBDG) to evaluate glucose uptake capacity [28]. By using antibodies against surface markers CD133 and EpCAM, the putative T-IC subsets were distinguished from the bulk tumor cells, and their fluorescence intensity that represented the uptake of glucose was determined by flow cytometric analysis 
(Fig. 2C). In comparison to the non-TIC counterparts, the liver T-IC subpopulation showed a much higher competence in glucose uptake as demonstrated by elevated 2-NBDG fluorescence in CD133- or EpCAM-positive HCC cells (Fig. 2D). Next, we compared the uptake of glucose in different primary HCC cell subpopulations. As expected, both isolated CD133- and EpCAM-positive cells exhibited much higher 2-NBDG uptake than their matched non-TIC fractions in bulk tumor cells (Fig. 2E). In short, these findings suggested that liver T-ICs can outcompete their non-TIC counterparts by preferentially taking up glucose and thus surviving the restricted glucose conditions.

\subsection{GLUT1 and GLUT3 expressions partially contributed to the preferential glucose} uptake in liver T-ICs.

Glucose uptake in malignant tumors mainly relies on the presence of glucose transporters, namely, the Glut family of proteins [29]. To address whether the differential expression of Glut isoforms in liver T-ICs contributed to preferential glucose uptake, we examined the mRNA levels of GLUT isoforms using qRT-PCR analysis. As shown in Fig. 3A and Fig. 3B, the mRNA levels of two Glut isoforms (GLUT1 and GLUT3) were upregulated in $\mathrm{CD}_{133^{+}}$cells that were sorted from HCC cell lines and primary HCC cells and compared with their matched CD133 cells. In addition, although the mRNA levels of GLUT2 and GLUT4 were consistent but much more modestly increased in CD133 ${ }^{+}$T-ICs, the differences did not reach statistical significance (Fig. 3A, B). We also evaluated the GLUTs expression in our model via Western blot assay. Protein levels of GLUT1 and GLUT3 were consistently elevated in T-ICs when compared with those non-TICs, but GLUT2 and GLUT4 contents were almost not changed between T-ICs and non-TICs (Fig. 3C). Next, we used small interfering RNAs 
(siRNA) to knock down the expression of GLUT1 and GLUT3, and the silencing effects were verified by qRT-PCR assay (Fig. 3D). It seemed that neither silencing of GLUT1 nor GLUT3 altered the glucose uptake capacity of those non-TIC subsets. However, the 2-NBDG uptake was notably reduced in the sorted CD133-expressing cells (Fig. 3E). These findings implied that Glut isoforms were differentially expressed in HCCs and might potentially influence the glucose uptake capacities of liver T-ICs. It is well established that the transcription factors such as OCT4, SOX2, and NANOG are regarded as the core circuits of stem cells in the tumor hierarchy [30]. This notion was confirmed by our data that the mRNA levels of these "stemness" markers, in particular OCT4, were significantly elevated in sorted CD133 ${ }^{+} \mathrm{HCC}$ cells (Fig. 3F). It has been proven that OCT4 activation plays a critical role in tumorigenesis by driving the self-renewal of tumor T-ICs [31]. On the basis of these observations, we evaluated the transcript levels of the GLUTS and OCT4 in clinical HCC tissues. As expected, the expression levels of GLUT1 and GLUT3 were correlated well with OCT4 in HCC samples. However, there was no obvious relevance between OCT4 and GLUT2 or GLUT4 (Fig. 3G). Together, these data suggested that specific Glut isoforms could potentially enhance the glucose uptake in liver T-ICs.

\subsection{Inhibition of Gluts-mediated glucose uptake attenuated the stem-like properties of}

\section{liver T-ICs.}

To determine the role of specific Glut isoforms in the maintenance of T-ICs, we used GLUT inhibitor Phloretin (Phl) to block the uptake of glucose [32],[33]. After exposure to Phl treatment, the percentage of $\mathrm{CD}_{133^{+}}$T-ICs was significantly decreased in Huh7 and MHCC-97L cells (Fig. 4A). Moreover, HCC cells transfected with siRNA targeting GLUT1 
or GLUT3 displayed a decreased percentage in the $\mathrm{CD}_{133^{+}}$population compared with cells expressing control siRNA (Fig. 4B). These results pinpointed the importance of Gluts-mediated glucose uptake in maintaining T-IC subpopulations. Liver T-ICs are capable of forming tumor spheroids in vitro, which is a phenotype associated with self-renewal competence [7]. Herein, we performed a tumor spheroid formation assay to substantiate the influence of glucose uptake inhibition on the biological characteristics of liver T-ICs. As shown in Fig. 4C, Phl treatment resulted in a dramatic reduction in size and number of tumor spheroids in CD133 ${ }^{+}$MHCC-97L and Huh7 cells. Similar results were also obtained in sorted $\mathrm{CD}_{133^{+}}$primary liver cancer cell pools (Fig. 4D). More importantly, knockdown of GLUT1 or GLUT3 also attenuated the spheroid formation potential of $\mathrm{CD}_{133^{+}} \mathrm{HCC}$ subsets in condition of low glucose (Fig. 4E). Steply, we also investigated the whole effects in vitro and in vivo when both GLUT1 and GLUT3 were blocked. As shown in Fig. 4F and G, the concomitant transfection of shRNAs targeting GLUT1 and GLUT3 achieved significant knockdown effects in Huh7 cells. Interestingly, silencing of both GLUT1 and GLUT3 not only ultimately prevented sphere formation in condition of low glucose in vitro but also led to a significant decrease in size and number of tumor formation in vivo (Fig. 4H-4I). Together, our data indicated that GLUTs-mediated glucose uptake was required for the maintenance of liver T-ICs.

In current study, we also speculate that if the increased glucose uptake subsequently enhanced metabolic pathways such as mitochondrial respiration, lipid metabolism, glutamine metabolism, or pentose phosphate metabolism responsible for metabolic reprogramming in liver T-ICs in our cancer model. To verify our hypothesis, we detected the expression levels of multiple metabolic enzymes which usually catalyze the rate-limiting reactions in glycolysis-related metabolic pathways [34]. For instance, phosphoglycerate dehydrogenase 
(PHGDH) and Glucose-6-phosphate dehydrogenase (G6PD) catalyze the committed steps in the serine biosynthetic pathway and pentose phosphate pathway (PPP), respectively [14]; Peroxisome proliferator-activated receptor gamma coactivator 1 -alpha (PGC-1 $\alpha$ ) is a master regulator of mitochondria biogenesis [35]; Glutaminase (GLS) is a key enzyme that plays an essential role in the catabolism of glutamine [36]; Both Fatty acid synthase (FASN) and Acetyl-CoA carboxylase (ACACA) mainly catalyze de novo fatty acid synthesis, whereas carnitine palmitoyltransferase 1 (CPT1A) represents the activation of fatty acid oxidation process [37]. As shown in Supplementary figure 1, the protein levels of PHGDH, G6PD, and PGC- $1 \alpha$ were up-regulated in $\mathrm{CD}_{133^{+}} \mathrm{T}$-ICs under glucose starvation. In contrast, the GLS content was somewhat decreased in T-ICs after glucose deprivation. Additionally, the expression levels of FASN, ACACA and CPT1A were not changed between T-ICs and non-TICs even in low glucose culture conditions. Taken together, our findings indicate that liver T-ICs may enhance glucose uptake and glycolytic-related pathways including serine biosynthesis, PPP and mitochondrial respiration to promote cell survival under low glucose.

\subsection{IL-6/STAT3 signaling regulated Gluts-mediated glucose uptake in liver T-ICs.}

We next explored the underlying mechanisms responsible for the differential Gluts expression and preferential glucose uptake in liver T-ICs. A series of well-documented signaling pathways including $\mathrm{HGF} / \mathrm{c}-\mathrm{Met}$, EGFR, IGF/insulin, $\beta$-catenin/Wnt, and IL-6/STAT3have been found to promote T-IC phenotypes and "stemness" properties [38],[39]. Hence, we wondered whether the activation of these TIC-related signaling pathways affected glucose uptake. To address this issue, several growth factors (HGF, EGF, and IGF), interleukin-6 (IL-6), and the agonist targeting the Wnt pathway (Wnt3a) were applied to activate these "stemness"-related signal pathways in both the T-IC subset and their non-TIC counterparts. Interestingly, we observed that both Wnt3a and IL-6 stimulation could increase the uptake of $2-\mathrm{NBDG}$ in the $\mathrm{CD}_{133^{+}}$cell pool (Fig. 5A). IL-6 induced a much higher elevation of 2-NBDG uptake than Wnt3a in CD133 ${ }^{+}$T-ICs, particularly under restricted 
glucose. In contrast, the 2-NBDG fluorescence was only slightly elevated in CD133- $\mathrm{HCC}$ cells after exposure to Wnt3a and IL-6 either under normal or limited glucose(Fig. 5A), further implying the importance of the IL-6/STAT3 signaling pathway in regulating the glucose uptake capacity of the T-IC subpopulations. Next, we pharmacologically inhibited the IL-6/STAT3 pathway by using S3I-201 and WP1066 as previously reported [40],[41]. The targeting of the STAT3 pathway decreased the uptake of 2-NBDG in CD133 ${ }^{+}$T-ICs under glucose deprivation, but no significant effect was observed on their matched non-TICs subset (Fig. 5B). Meanwhile, inhibition of the STAT3 pathway largely abolished the survival advantage of $\mathrm{CD}_{133^{+}}$HCC cells under glucose deprivation (Fig. 5C), further indicating the requirement for IL-6/STAT3-mediated glucose uptake in liver stem-like cells. Considering that STAT3 is directly activated by IL-6 through phosphorylation of tyrosine 705 , and it acts as a transcription activator [42], we intended to test if STAT3 could affect the transcriptional activity of GLUTs in HCCs. RT-PCR assays revealed that IL-6 stimulation markedly upregulated the transcriptional levels of GLUT1 and GLUT3 (Fig. 5D), indicating that the activation of the IL-6/STAT3 pathway could promote transcriptional activities of Glut isoforms and thus facilitate glucose uptake capacities.

3.6 Inhibition of glucose uptake sensitized liver T-ICs to sorafenib treatment and enhanced the therapeutic efficacy in vivo.

Anti-angiogenic agents are currently used as adjuvant therapies for cancer management [43]. The multi-kinase inhibitor sorafenib is the only drug approved for the treatment of non-resectable HCCs [44]. However, high rates of acquired drugresistance severely limit its clinical application [45]. We first observed that sorafenib treatment obviously enhanced 
glucose uptake and lactate generation in HCC cells, whereas Phl administration could partially abolish this effect (Fig. 6A, B). These results indicated that the glycolytic pathway in HCCs was strengthened in the presence of sorafenib. Furthermore, we administered sorafenib to mice xenotransplanted with an HCC cell line (Huh7) and used small animal PET/CT scans with ${ }^{18} \mathrm{~F}$-fluorodeoxyglucose $\left({ }^{18} \mathrm{~F}\right.$-FDG) to evaluate the effect of sorafenib on glucose uptake in HCCs in vivo. Interestingly, PET images showed that ${ }^{18} \mathrm{~F}$-FDG uptake was rarely detected in the core area but had a tendency to increase in the peripheral regions of the sorafenib-treated xenografts (Fig. 6C). This phenomenon may be due to the anti-angiogenesis effect of sorafenib, which led to the extensive necrotic cell death in the inner region of the HCC nodules. Moreover, sorafenib treatment enhanced glucose uptake capacity of HCCs in vivo, as revealed by the higher levels of ${ }^{18} \mathrm{~F}-\mathrm{FDG}$ uptake in sorafenib-treated HCCs compared to xenografts treated with saline (Fig. 6D). These findings further support the notion that sorafenib could increase the glucose uptake of HCCs. Next, we explored the influence of sorafenib treatment in the maintenance of liver T-ICs. Flow cytometry revealed that the percentage of $\mathrm{CD}_{133^{+}}$cells in sorafenib-treated xenografts was much higher than in the control group (Fig. 6E). More interestingly, sorafenib administration remarkably elevated the glucose uptake capacity of $\mathrm{CD}_{133^{+}}$cells compared to their counterparts (Fig. 6F). Further investigation showed that sorafenib treatment selectively enhanced protein and mRNA expression of GLUT1 and GLUT3 in T-ICs isolated from mouse model (Fig. 6G and 6H). Taken together, our data suggest that sorafenib treatment induces the expansion of putative T-ICs and preferentially enhances their glucose uptake ability.

Next, we investigated the potential role of glucose in the maintenance of 
sorafenib-induced T-IC subsets in vitro. To address this issue, HCC cells were incubated in standard medium or starved in the presence of sorafenib for 3 days, and the proportion of $\mathrm{CD}_{133^{+}}$cells in the total cell pool was assessed. In agreement with the previous in vivo results, sorafenib treatment enriched the putative T-IC subpopulations under standard culture conditions, whereas limited glucose decreased the populations of $\mathrm{CD}_{133^{+}} \mathrm{T}$-ICs upon sorafenib treatment (Fig. 7A). Consistently, the cell apoptosis assay confirmed that the fraction of T-ICs were more sensitive to sorafenib under low-glucose conditions (Fig. 7B). Based on the aforementioned results, we wondered whether targeting glycolysis could potentiate the anti-tumor effects of sorafenib in HCC cells, especially the fraction of T-ICs. Treatment of Huh7 cells with sorafenib or Phl alone reduced cell survival. Remarkably, the combination of $\mathrm{Phl}$ and sorafenib significantly decreased cell viability, implying that the glucose uptake inhibitor effectively enhanced sorafenib-induced cell death in hepatoma cells (Fig. 7C). Apoptosis assay showed that Phl treatment significantly promoted sorafenib-stimulated cell apoptosis in hepatoma cells (Fig. 7D). Additionally, we further confirmed this synergetic effect of cellkilling in nude mice bearing CD133 ${ }^{+}$Huh7 cell-derived xenografts. Consistent with the in vitro results, treatment with $\mathrm{Phl}$ or sorafenib alone partially inhibited the growth of HCC tumor xenografts, whereas combined treatment significantly suppressed tumor progression (Fig. 7E and F). Taken together, these findings suggest that inhibition of glucose uptake can enhance the therapeutic efficacy of sorafenib in vivo and indicates the potential clinical utility of this regimen.

\section{Discussion}


Accumulating evidence has demonstrated that T-ICs are more resistant to routine chemotherapies, which may be responsible for tumor progression and relapse [46],[47]. These findings prompted us to explore the potential biological characteristics that endow the survival advantage of T-ICs in bulk tumor cells. To date, although reprogrammed metabolism has emerged as a hallmark of malignancies and plays an important role in cancer development [15], the metabolic alternations are still weakly linked to cancer cellular hierarchies, especially the cancer stem-like cells. Our current results suggested that the putative CD133 ${ }^{+}$T-ICs exhibited higher capacities for glucose uptake relative to their non-TIC counterparts. Furthermore, we observed that the glucose uptake competence in T-ICs was correlated with the expression of differential glucose transporters (GLUTs), which was at least in part mediated by the IL-6/STAT3 signaling axis. This specific metabolic phenotype allows liver T-ICs to compete successfully for available nutrients in scarcity and to survive under harsh microenvironments. Therefore, the targeting of GLUTs-mediated glucose uptake could be an effective anti-tumor strategy for the eradication of T-ICs.

Although the reliance of cancer cells on aerobic glycolysis is a commonly accepted metabolic phenotype, the metabolic alternations of CSCs remain a relatively "blank" area with limited investigation. Previous studies have shown that the CSCs from glioblastoma, leukemia, ovarian, and breast cancers relied more on oxidative phosphorylation as their primary source of energy production, whereas their differentiated counterparts generated energy largely through aerobic glycolysis [48],[49]. In contrast, Palorini and colleagues reported that CSCs derived from an osteosarcoma cell line presented a prominent glycolytic phenotype [50]. Similarly, such metabolic requirements were also observed in side-population cells from 
the non-small cell lung cancer cell line A549 [51]. Our present data suggested that enhanced glucose uptake was required for the survival and propagation of liver TIC subsets. We observed that liver T-IC subsets preferentially took up glucose and survived in limited glucose conditions. In agreement with our findings, another study addressing the metabolic profiles of brain T-ICs found that nutrient restriction enriched the populations of glioma stem cells, which could directly outcompete for limited glucose by upregulating the high affinity glucose transporter GLUT3 [23]. This notion was also supported by our observations that both GLUT1 and GLUT3 were preferentially expressed in liver T-IC subsets. Although we noted that GLUT2 was the predominant isoform expressed in HCC cells, GLUT1 and GLUT3 were differentially expressed in liver T-ICs and correlated with the "stemness" phenotype. Thus, these data indicated that upregulation of specific Gluts are required for T-ICs to sustain access to limited nutrients in a harsh environment.

We also explored the underlying mechanisms responsible for the upregulation of Glut proteins in T-ICs. Activated by the pro-inflammatory cytokine IL-6, STAT3 plays a critical role in inflammation-associated HCC tumorigenesis and progression [52]. Additionally, the IL-6/STAT3 signaling axis has been previously reported to be involved in the maintenance of liver CSCs [53],[10]. Recently, it has been found that IL-6/STAT3 signaling upregulated CD133 expression by interacting with NF- $\mathrm{BB}$ andHIF-1 $\alpha$ during $\mathrm{HCC}$ formation [54]. In addition to these findings, we provided data to show a novel regulatory effect of IL-6/STAT3 signaling on the metabolic phenotypes of T-ICs. Our results indicated that IL-6-induced STAT3 activation could promote Gluts expression in T-ICs and thus enhance their glucose uptake competency. Conversely, targeting the IL-6/STAT3 signaling axis by pharmacological inhibitors and 
siRNAs abolished this effect. Although it seems that the IL-6/STAT3 signaling pathway could enhance the transcriptional activity of GLUT1 and GLUT3, we are still not sure if IL-6/STAT3 could directly attach to the promoter regions of GLUTs. Therefore, the underlying mechanism by which STAT3 regulates GLUTs still needs further investigation. On the basis of these data, we believe that interfering with the STAT3-mediated GLUTs upregulation might represent a promising therapeutic strategy by eradicating the T-IC subpopulations.

Sorafenib is the first agent demonstrated to have a survival benefit for patients with advanced HCC [44],[55]. However, its therapeutic effect is heterogeneous and frequently transient due to drug resistance [55]. T-IC subsets in cancers are considered to be responsible for chemoresistance [56]. However, the influence exerted by external stimuli and tumor-related microenvironments on T-ICs are rarely taken into consideration. A recent study has revealed that sorafenib could enrich EpCAM-positive liver T-ICs and promote their stem-like properties via mediating the TCS2-AKT signaling pathway [57]. In agreement with their results, we found that sorafenib treatment enriched $\mathrm{CD} 133^{+} \mathrm{T}-\mathrm{IC}$ subpopulations through unknown mechanisms. Moreover, these cells were more resistant to sorafenib-induced cell death compared to non-TICs in bulk tumor cells. Here, we provide data to show that sorafenib treatment significantly facilitated the uptake of glucose and promoted the glycolytic phenotype particular to liver T-ICs, while the targeting of glucose uptake by Phl reversed these metabolic alternations. Therefore, the inhibition of glucose uptake may potentiate the therapeutic effect of sorafenib, and this combination could be a promising approach for HCC treatment.

Taken together, these findings represent the first time that IL-6 stimulation induced 
GLUTs expression in a STAT3-dependent manner, leading to enhanced glucose uptake in T-ICs and thus conferring these cells a survival advantage particularly under restricted glucose conditions. In summary, our findings provide not only a novel mechanism of IL-6/STAT3-mediated Gluts upregulation in liver T-ICs to initiate HCC but also a promising combined therapy regimen for HCC management.

\section{Acknowledgments}

The authors thank Pardeep Kumar for editing the manuscript. The authors also thank Shan-Hua Tang, Shan-Na Huang, Dong-Ping Hu and Dan-Dan Huang for their technical assistance.

\section{References}

1. Marrero JA. Hepatocellular carcinoma. Current opinion in gastroenterology 2006, 22(3):248-253.

2. Bruix J, Boix L, Sala M, et al. Focus on hepatocellular carcinoma. Cancer cell 2004, 5(3):215-219.

3. Tung-Ping Poon R, Fan ST, Wong J: Risk factors, prevention, and management of postoperative recurrence after resection of hepatocellular carcinoma. Ann Surg 2000, 232(1):10-24.

4. Aravalli RN, Steer CJ, Cressman EN. Molecular mechanisms of hepatocellular carcinoma. Hepatology 2008, 48(6):2047-2063.

5. Reya T, Morrison SJ, Clarke MF, et al. Stem cells, cancer, and cancer stem cells. Nature 2001, 414(6859):105-111.

6. Yang ZF, Ho DW, Ng MN, et al. Significance of CD90+ cancer stem cells in human liver cancer. Cancer cell 2008, 13(2):153-166.

7. Yamashita T, Ji J, Budhu A, et al. EpCAM-positive hepatocellular carcinoma cells are tumor-initiating cells with stem/progenitor cell features. Gastroenterology 2009, 136(3):1012-1024.

8. Jordan CT, Guzman ML, Noble M. Cancer stem cells. The New England journal of medicine 2006, 355(12):1253-1261.

9. $\mathrm{Ma} \mathrm{S}, \mathrm{Chan} \mathrm{KW}, \mathrm{Hu} \mathrm{L}$, et al. Identification and characterization of tumorigenic liver cancer stem/progenitor cells. Gastroenterology 2007, 132(7):2542-2556.

10. Lee TK, Castilho A, Cheung VC, et al. CD24(+) liver tumor-initiating cells drive self-renewal and tumor initiation through STAT3-mediated NANOG regulation. Cell stem cell 2011, 9(1):50-63. 
11. Yang W, Wang C, Lin Y, et al. OV6(+) tumor-initiating cells contribute to tumor progression and invasion in human hepatocellular carcinoma. Journal of hepatology 2012, 57(3):613-620.

12. Diehn M, Majeti R. Metastatic cancer stem cells: an opportunity for improving cancer treatment? Cell stem cell 2010, 6(6):502-503.

13. Warburg O. On respiratory impairment in cancer cells. Science 1956, 124(3215):269-270.

14. Vander Heiden MG, Cantley LC, Thompson CB: Understanding the Warburg effect: the metabolic requirements of cell proliferation. Science 2009, 324(5930):1029-1033.

15. Hanahan D, Weinberg RA. Hallmarks of cancer: the next generation. Cell 2011, 144(5):646-674.

16. Cairns RA, Harris IS, Mak TW. Regulation of cancer cell metabolism. Nature reviews Cancer 2011, 11(2):85-95.

17. Hsu PP, Sabatini DM. Cancer cell metabolism: Warburg and beyond. Cell 2008, 134(5):703-707.

18. Magee JA, Piskounova E, Morrison SJ. Cancer stem cells: impact, heterogeneity, and uncertainty. Cancer cell 2012, 21(3):283-296.

19. Hirayama A, Kami K, Sugimoto M, et al. Quantitative metabolome profiling of colon and stomach cancer microenvironment by capillary electrophoresis time-of-flight mass spectrometry. Cancer research 2009, 69(11):4918-4925.

20. Gullino PM, Grantham FH, Courtney $\mathrm{AH}$, et al. Relationship between oxygen and glucose consumption by transplanted tumors in vivo. Cancer research 1967, 27(6):1041-1052.

21. Li Z, Bao S, Wu Q, et al. Hypoxia-inducible factors regulate tumorigenic capacity of glioma stem cells. Cancer cell 2009, 15(6):501-513.

22. Wang Y, Liu Y, Malek SN, et al. Targeting HIF1alpha eliminates cancer stem cells in hematological malignancies. Cell stem cell 2011, 8(4):399-411.

23. Flavahan $\mathrm{WA}, \mathrm{Wu} \mathrm{Q}$, Hitomi $\mathrm{M}$, et al. Brain tumor initiating cells adapt to restricted nutrition through preferential glucose uptake. Nature neuroscience 2013, 16(10):1373-1382.

24. Wang MD, Wu H, Fu GB, et al. Acetyl-CoA carboxylase alpha promotion of glucose-mediated fatty acid synthesis enhances survival of hepatocellular carcinoma in mice and patients. Hepatology 2015.

25. Urasaki Y, Heath L, Xu CW. Coupling of glucose deprivation with impaired histone H2B monoubiquitination in tumors. PloS one 2012, 7(5):e36775.

26. Dwarakanath BS. Cytotoxicity, radiosensitization, and chemosensitization of tumor cells by 2-deoxy-D-glucose in vitro. Journal of cancer research and therapeutics 2009, 5 Suppl 1:S27-31.

27. Bhardwaj R, Sharma PK, Jadon SS, et al. A combination of 2-deoxy-D-glucose and 6-aminonicotinamide induces oxidative stress mediated selective radiosensitization of malignant cells via mitochondrial dysfunction. Tumour biology : the journal of the International Society for Oncodevelopmental Biology and Medicine 2011, 32(5):951-964.

28. Yoshioka K, Takahashi H, Homma T, et al. A novel fluorescent derivative of glucose applicable to the assessment of glucose uptake activity of Escherichia coli. Biochimica et biophysica acta 1996, 1289(1):5-9.

29. Macheda ML, Rogers S, Best JD. Molecular and cellular regulation of glucose transporter (GLUT) proteins in cancer. Journal of cellular physiology 2005, 202(3):654-662.

30. Kumar SM, Liu S, Lu H, et al. Acquired cancer stem cell phenotypes through Oct4-mediated dedifferentiation. Oncogene 2012, 31(47):4898-4911.

31. Zhu P, Wang Y, He L, et al. ZIC2-dependent OCT4 activation drives self-renewal of human liver cancer stem cells. J Clin Invest 2015, 125(10):3795-3808.

32. Wu CH, Ho YS, Tsai $\mathrm{CY}$, et al. In vitro and in vivo study of phloretin-induced apoptosis in human liver 
cancer cells involving inhibition of type II glucose transporter. International journal of cancer Journal international du cancer 2009, 124(9):2210-2219.

33. Tomioka S. Water transport by glucose transporter type 3 expressed in Xenopus oocytes. Neuroreport 2012, 23(1):21-25.

34. Boroughs LK, DeBerardinis RJ. Metabolic pathways promoting cancer cell survival and growth. Nature cell biology 2015, 17(4):351-359.

35. Vazquez F, Lim JH, Chim H, et al. PGC1alpha expression defines a subset of human melanoma tumors with increased mitochondrial capacity and resistance to oxidative stress. Cancer cell 2013, 23(3):287-301.

36. Son J, Lyssiotis CA, Ying $H$, et al. Glutamine supports pancreatic cancer growth through a KRAS-regulated metabolic pathway. Nature 2013, 496(7443):101-105.

37. Carracedo A, Cantley LC, Pandolfi PP. Cancer metabolism: fatty acid oxidation in the limelight. Nat Rev Cancer 2013, 13(4):227-232.

38. De Luca A, Fiorillo $M$, Peiris-Pages $M$, et al. Mitochondrial biogenesis is required for the anchorage-independent survival and propagation of stem-like cancer cells. Oncotarget 2015, 6(17):14777-14795.

39. Pang RW, Poon RT. From molecular biology to targeted therapies for hepatocellular carcinoma: the future is now. Oncology 2007, 72 Suppl 1:30-44.

40. Bu LL, Zhao ZL, Liu JF, et al. STAT3 blockade enhances the efficacy of conventional chemotherapeutic agents by eradicating head neck stemloid cancer cell. Oncotarget 2015, 6(39):41944-41958.

41. Zhou X, Ren Y, Liu A, et al. WP1066 sensitizes oral squamous cell carcinoma cells to cisplatin by targeting STAT3/miR-21 axis. Scientific reports 2014, 4:7461.

42. Yuan ZL, Guan YJ, Wang L, et al. Central role of the threonine residue within the $p+1$ loop of receptor tyrosine kinase in STAT3 constitutive phosphorylation in metastatic cancer cells. Molecular and cellular biology 2004, 24(21):9390-9400.

43. Fernandez M, Semela D, Bruix J, et al. Angiogenesis in liver disease. Journal of hepatology 2009, 50(3):604-620.

44. Llovet JM, Ricci S, Mazzaferro V, et al. Sorafenib in advanced hepatocellular carcinoma. The New England journal of medicine 2008, 359(4):378-390.

45. Gusani NJ, Jiang Y, Kimchi ET, et al. New pharmacological developments in the treatment of hepatocellular cancer. Drugs 2009, 69(18):2533-2540.

46. Dean M, Fojo T, Bates S. Tumour stem cells and drug resistance. Nat Rev Cancer 2005, 5(4):275-284.

47. Singh A, Settleman J. EMT, cancer stem cells and drug resistance: an emerging axis of evil in the war on cancer. Oncogene 2010, 29(34):4741-4751.

48. Vlashi E, Lagadec C, Vergnes L, et al. Metabolic state of glioma stem cells and nontumorigenic cells. Proceedings of the National Academy of Sciences of the United States of America 2011, 108(38):16062-16067.

49. Lagadinou ED, Sach A, Callahan K, et al. BCL-2 inhibition targets oxidative phosphorylation and selectively eradicates quiescent human leukemia stem cells. Cell stem cell 2013, 12(3):329-341.

50. Palorini R, Votta G, Balestrieri C, et al. Energy metabolism characterization of a novel cancer stem cell-like line 3AB-OS. Journal of cellular biochemistry 2014, 115(2):368-379.

51. Liu PP, Liao J, Tang ZJ, et al. Metabolic regulation of cancer cell side population by glucose through activation of the Akt pathway. Cell death and differentiation 2014, 21(1):124-135.

52. Turkson J, Jove R. STAT proteins: novel molecular targets for cancer drug discovery. Oncogene 2000, 
19(56):6613-6626.

53. Tang $\mathrm{Y}$, Kitisin $\mathrm{K}$, Jogunoori $\mathrm{W}$, et al. Progenitor/stem cells give rise to liver cancer due to aberrant TGF-beta and IL- 6 signaling. Proceedings of the National Academy of Sciences of the United States of America 2008, 105(7):2445-2450.

54. Won $\mathrm{C}, \mathrm{Kim} \mathrm{BH}, \mathrm{Yi} \mathrm{EH}$, et al. Signal transducer and activator of transcription 3-mediated CD133 up-regulation contributes to promotion of hepatocellular carcinoma. Hepatology 2015, 62(4):1160-1173.

55. Cheng AL, Kang YK, Chen Z, et al. Efficacy and safety of sorafenib in patients in the Asia-Pacific region with advanced hepatocellular carcinoma: a phase III randomised, double-blind, placebo-controlled trial. The Lancet Oncology 2009, 10(1):25-34.

56. Dalerba P, Cho RW, Clarke MF. Cancer stem cells: models and concepts. Annual review of medicine 2007, 58:267-284.

57. Guan DX, Shi J, Zhang Y, Zhao JS, Long LY, Chen TW, Zhang EB, Feng YY, Bao WD, Deng YZ et al: Sorafenib enriches epithelial cell adhesion molecule-positive tumor initiating cells and exacerbates a subtype of hepatocellular carcinoma through TSC2-AKT cascade. Hepatology 2015, 62(6):1791-1803.

\section{Figure Legends}

Figure 1: Enriched liver T-ICs preferentially survive under restricted glucose conditions.

A. MHCC-97L and Huh7 cells were maintained in cultural media containing standard $(25 \mathrm{mM})$ or restricted $(2.5 \mathrm{mM})$ concentrations of glucose for $48 \mathrm{hr}$. The percentage of CD133-expressing cells in the total cell pools was detected using flow cytometry analysis. The data shown (left part) are representative of three independent experiments $(* \mathrm{p}<0.05$; **p < 0.01). B. Flow cytometry analysis of EpCAM-expressing HCC cells incubated under complete media or a low-glucose condition $\left(2.5 \mathrm{mM}\right.$ glucose) for $48 \mathrm{hr}$ is shown $\left({ }^{*} \mathrm{p}<0.05\right.$; $* * \mathrm{p}<0.01$, and $* * * \mathrm{p}<0.001)$. C. The indicated HCC cells were incubated under complete media with or without 2-DG $(5 \mathrm{mM}$, or $10 \mathrm{mM})$ treatment for $48 \mathrm{hr}$. The proportion of $\mathrm{CD}_{133^{+}}$HCC cells was assessed using flow cytometry. D. Flow cytometric analysis of 
representative samples of primary hepatoma cells from 3 different patients with HCC is shown (Case No.: \#1, \#2, and \#3). Isolated primary HCC cells were maintained in a low-glucose condition $(2.5 \mathrm{mM})$ or in complete media supplemented with $10 \mathrm{mM} 2$-DG for $48 \mathrm{hr}$. Percentages of $\mathrm{CD}_{133^{+}}$(left panel) or $\mathrm{EpCAM}^{+}$(right panel) cells in total cell pools were detected by flow cytometry $(* \mathrm{p}<0.05, * * \mathrm{p}<0.01$, and $* * * \mathrm{p}<0.001)$. All experiments were performed in triplicate, and data are shown as means \pm SD.

Figure 2: Liver T-ICs exhibit elevated glucose uptake competence. A-B. Annexin V/Propidium Iodide (PI) staining was used to evaluate cell apoptosis of CD133 ${ }^{+}$T-ICs and non-T-IC subsets after culture in low glucose $(2.5 \mathrm{mM})$ for $48 \mathrm{hr}$. Flow cytometry scatter plots are shown and the percentages of apoptotic cells were quantified $(* * * p<0.001)$. C. An overlay of representative flow plots is shown. MHCC-97L cells were labeled with the fluorescent glucose analog (2-NBDG) for $45 \mathrm{~min}$. The proportion of $\mathrm{CD} 133^{+}$cells was assessed (left panel), and the uptake of fluorescent 2-NBDG in CD133-sorted T-ICs (the fraction marked green) or non-T-ICs (cell populations marked in black) was determined by flow cytometry (right panel). D. Flow cytometry demonstrates that 2-NBDG uptake is higher in CD133 or EpCAM-expressing HCC cells under standard cultural conditions $(* * \mathrm{p}<0.01$, $* * * \mathrm{p}<0.001)$. E. The uptake of 2-NBDG in T-ICs or their matched non-TICs isolated from clinical HCC specimens (Case No.: \#1, \#2, and \#3) was detected using flow cytometry (*p < $0.05, * * \mathrm{p}<0.01$, and $* * * \mathrm{p}<0.001)$. All experiments were performed in triplicate, and data are shown as means $\pm \mathrm{SD}$. 
Figure 3: GLUT1 and GLUT3 expressions partially contribute to the preferential glucose uptake in liver T-ICs. A-B. Quantitative real-time PCR (qRT-PCR) was used to measure the mRNA expressions of glucose transporters GLUT1-4 in CD133-sorted T-ICs and their matched non-T-ICs isolated from unenriched HCC cell lines (MHCC-97L and Huh7 cells) or clinical HCC tissue under normal cultural medium (**p $<0.01$,*** $p<0.001)$. C. The protein levels of GLUT1-4 in CD133-sorted T-ICs and their matched non-T-ICs isolated from HCC cell lines or clinical HCC samples were detected by western blot assay. $\beta$-actin was used as internal loading control. D. qRT-PCR analysis was performed for GLUT1 and GLUT3 in sorted $\mathrm{CD} 133^{+}$Huh7 cells transfected with siRNAs targeting GLUT1 and GLUT3, respectively $(* * * p<0.001)$. E. Flow cytometric analysis was performed to quantify the 2-NBDG uptake of CD133 ${ }^{+}$T-ICs and non-T-IC subsets that were magnetic sorted from Huh7 cells expressing siNC, siGlut1, and siGlut3 $(* * \mathrm{p}<0.01)$. F. The mRNA levels of OCT4, SOX2, and NANOG in CD133-expressing T-ICs and non-T-ICs isolated from indicated HCC cells were measured using RT-PCR analysis $\left(* \mathrm{p}<0.05,{ }^{* * *} \mathrm{p}<0.001\right)$. G. The correlations between OCT4 and GLUTs (1-4) expression in 87 cases of human primary HCC specimens were analyzed. All experiments were performed in triplicate, and data are shown as means \pm SD.

Figure 4: Inhibition of Gluts-mediated glucose uptake attenuates stem-like properties of liver T-ICs. A. Unenriched HCC cells (MHCC-97L and Huh7) were incubated in complete media in the presence or absence of Phl treatment for $24 \mathrm{hr}$. Next, flow cytometric assay was used to quantify the percentage of CD133-expressing cells in the total cell pool (*p < 0.05). B. 
Flow cytometric analysis was performed to quantify the proportion of $\mathrm{CD} 133^{+}$cells in Huh7 cells transfected with siRNAs targeting GLUT1 and GLUT3, respectively (*p < 0.05). C-D. Tumor spheroid formation analysis demonstrated that Phl treatment suppressed the formation of tumor spheroids, which were initiated by $\mathrm{CD} 133^{+} \mathrm{HCC}$ cells isolated from both HCC cell lines and primary HCC specimens (Case No.: \#5878 and \#5825). Representative images of HCC spheroids derived from $\mathrm{CD}_{133^{+}}$cells with or without $\mathrm{Phl}$ treatment are shown (left panel), and spheroid numbers were counted (right panel) $(* \mathrm{p}<0.05, * * p<0.01)$. E. Tumor spheroid formation of $\mathrm{CD}_{133^{+}} \mathrm{T}$-IC subsets that were magnetic sorted from Huh7 or MHCC-97L cells expressing siNC, siGLUT1, and siGLUT3. Representative images of HCC spheroids derived from $\mathrm{CD}_{133^{+}}$cells are shown (left panel), and spheroid numbers were counted (right panel) $(* p<0.05, * * p<0.01)$. F-G. The mRNA and protein levels of GLUTs in Huh7 cells expressing shCtrl or both shGLUT1 and shGLUT3 were detected by qRT-PCR and western blot assays, respectively. H. formation of CD133 ${ }^{+}$T-ICs sorted from Huh7 cells expressing shCtrl or both shGLUT1 and shGLUT3 were used to generate tumor spheroid. Representative images of HCC spheroids are shown (left panel), and spheroid numbers were counted (right panel) $(* * \mathrm{p}<0.01, * * * \mathrm{p}<0.001)$. I. Representative subcutaneous tumors from mice injected with $1 \times 10^{6}$ indicated lentivirus-infected CD133 ${ }^{+}$T-ICs after 4 weeks of transplantation are shown. All experiments were performed in triplicate, and data are shown as means $\pm \mathrm{SD}$.

Figure 5: IL6/STAT3 signaling regulates Gluts-mediated glucose uptake in liver T-ICs. A. Huh7 cells were incubated under complete medium or low-glucose conditions $(2.5 \mathrm{mM}$ 
glucose) in the presence of HGF (20 ng/mL), EGF (20 ng/mL), IGF (100 ng/mL), Wnt3a (100 $\mathrm{ng} / \mathrm{mL})$, or IL-6 (100 ng/mL) for $24 \mathrm{hr}$. Next, the flow cytometric analysis was performed to quantify the 2-NBDG uptake of both CD133+ ${ }^{+}$T-ICs and non-T-IC subsets that were magnetic sorted from different cell factor-treated Huh7 cells $(* \mathrm{p}<0.05, * * \mathrm{p}<0.01$, and $* * * \mathrm{p}<0.001)$. B. Huh7 cells were incubated under low-glucose conditions (2.5mM glucose) and treated with $50 \mu \mathrm{M}$ S3I-201 or $5 \mu \mathrm{M}$ WP1066 for $24 \mathrm{hr}$. Next, the 2-NBDG uptake in CD133 ${ }^{+}$T-ICs and non-TIC subsets isolated from inhibitor-treated Huh7 cells was measured using flow cytometry $(* \mathrm{p}<0.05)$. C. A cell apoptosis assay was performed to quantify the cell apoptosis of CD133 ${ }^{+}$T-ICs and non-T-IC subsets after culture in low glucose $(2.5 \mathrm{mM})$ supplemented with $50 \mu \mathrm{M}$ S3I-201 or $5 \mu \mathrm{M}$ WP1066 for $48 \mathrm{hr}$. Flow cytometry scatter plots are shown (left pannel) and the percentages of apoptotic cells were quantified $(* * p<0.01)$. D. The qRT-PCR analysis was performed for GLUT1-4 in CD133-expressing T-ICs isolated from unenriched Huh7 cells treated with IL-6 $(100 \mathrm{ng} / \mathrm{mL})$ or a control vehicle for $24 \mathrm{hrs}\left({ }^{*} \mathrm{p}<0.05\right)$. All experiments were performed in triplicate and data are shown as means \pm SD.

Figure 6: Sorafenib promotes glucose uptake of HCCs and expands T-IC subpopulations.

A. Flow cytometric analysis of 2-NBDG uptake in HCC cell lines (MHCC-97L and Huh7) treated with indicated reagents for $24 \mathrm{hr}$ is shown $\left(* \mathrm{p}<0.05,{ }^{*} \mathrm{p}<0.01\right)$. DMSO was used as a control vehicle. B. HCC cells were maintained in the complete medium and treated with DMSO, sorafenib (2 mM), Phl (1 $\mu \mathrm{M})$, or sorafenib plus Phl for $24 \mathrm{hr}$, respectively. Lactate concentration in the medium was detected after incubation. The lactate production was normalized by cell number at the end of the experiment $(* \mathrm{p}<0.05$, **p $<0.01)$. All 
experiments were performed in triplicate, and data are shown as means \pm SD. C. Representative coronal PET and fusion PET/CT sections show ${ }^{18}$ F-FDG uptake in mice bearing Huh7 cell-derived HCC xenografts. Mice were treated with sorafenib or the control vehicle, and tumors on the right flank are indicated by white arrows. D. Comparison of the standardized ${ }^{18} \mathrm{~F}$-FDG uptake value $\left(\mathrm{SUV}_{\text {mean }}\right)$ at 30 minutes post-injection in sorafenib or vehicle-treated HCC xenografts is shown. E. Primary HCC cells were isolated from Huh7 cell-derived HCC xenografts in sorafenib or the vehicle-treated group $(n=4)$. The percentage of $\mathrm{CD}_{133^{+}}$cells in the total cell pool was compared $(\mathrm{p}=0.027)$. F. The uptake values of 2-NBDG in $\mathrm{CD}_{133^{+}} \mathrm{T}$-ICs or their matched non-TICs isolated from sorafenib or vehicle-treated xenografts were detected using flow cytometry $\left({ }^{*} \mathrm{p}<0.05\right)$. G-H. The protein and mRNA levels of GLUT1-4 in CD133-sorted T-ICs isolated from Huh7 cell-derived HCC xenografts treated with vehicle or sorafenib were detected by western blot and qRT-PCR assays, respectively. $\beta$-actin was used as internal loading control. All data are shown as means \pm SD.

Figure 7: Inhibition of glucose uptake sensitizes liver T-ICs to sorafenib treatment and enhances the therapeutic efficacy in vivo. A. The percentage of CD133 ${ }^{+} \mathrm{HCC}$ cells (Huh7 and MHCC-97L) after being cultured under normal or limited-glucose $(2.5 \mathrm{mM})$ conditions with DMSO or sorafenib $(2 \mathrm{mM})$ treatment for 3 days is shown $(* \mathrm{p}<0.05$, **p $<0.01)$. B. Cell apoptosis analysis of CD133 ${ }^{+}$T-ICs and non-TIC subsets after culture in standard or low glucose $(2.5 \mathrm{mM})$ medium with or without sorafenib for $48 \mathrm{hr}$ is shown, and the percentages of apoptotic cells were quantified $(* * p<0.01)$. C. MHCC-97L cells after treatment with 
DMSO, sorafenib (2 mM), Phl $(1 \mu \mathrm{M})$, or sorafenib plus Phl for $48 \mathrm{hr}$. Representative microscopic images are shown. D. Cell apoptosis analysis of HCC cells were treated with indicted reagents for $48 \mathrm{hr}$ and then cell apoptosis analysis was performed using flow cytometric assay. Representative flow cytometry scatter plots are shown (left panel) and the percentages of apoptotic cells were quantified $(* * p<0.01, * * * p<0.001)$. E-F. Mice injected with sorted CD133 ${ }^{+}$Huh7 cells were treated with a saline vehicle, sorafenib, Phl, or sorafenib in combination with $\mathrm{Phl}$, and representative subcutaneous tumors after 30 days of transplantation. Tumor growth from each group was evaluated at the indicated time points, and all values are presented as means $\pm \mathrm{SD}(* \mathrm{p}<0.05, * * \mathrm{p}<0.01)$ 
A

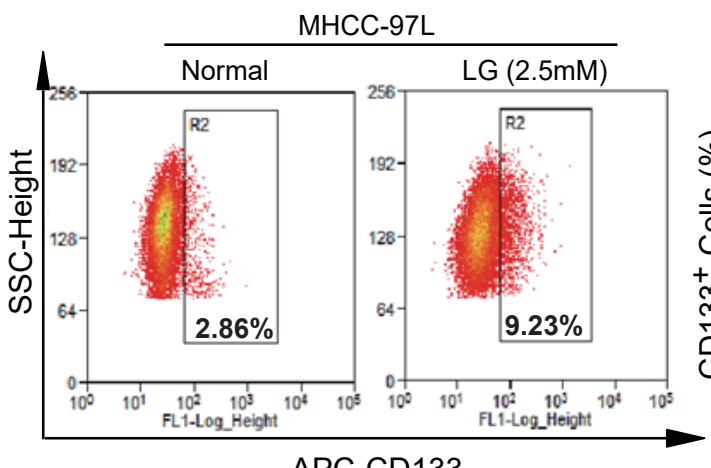

MHCC-97L
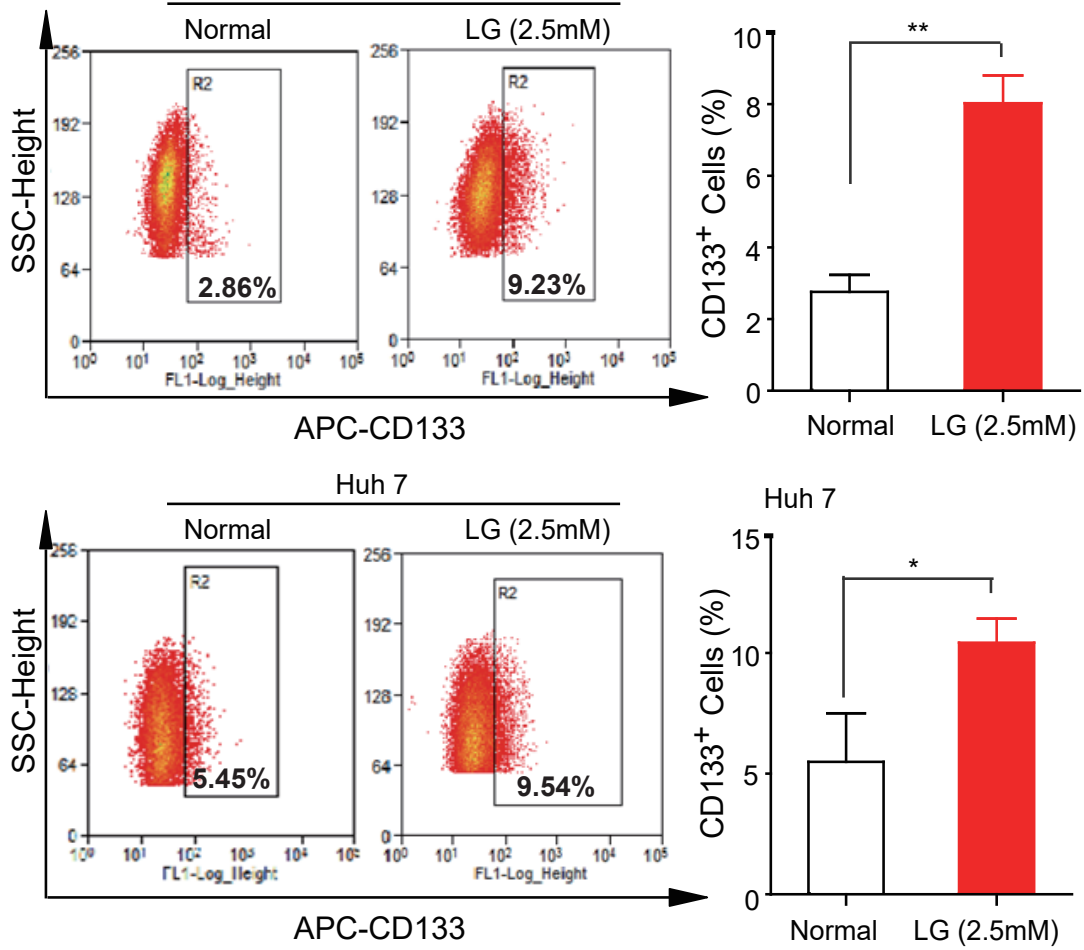

D

Primary HCC cells

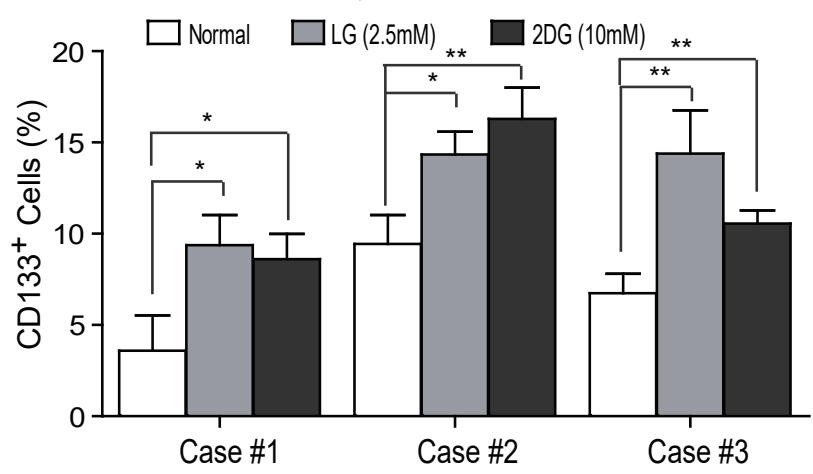

B
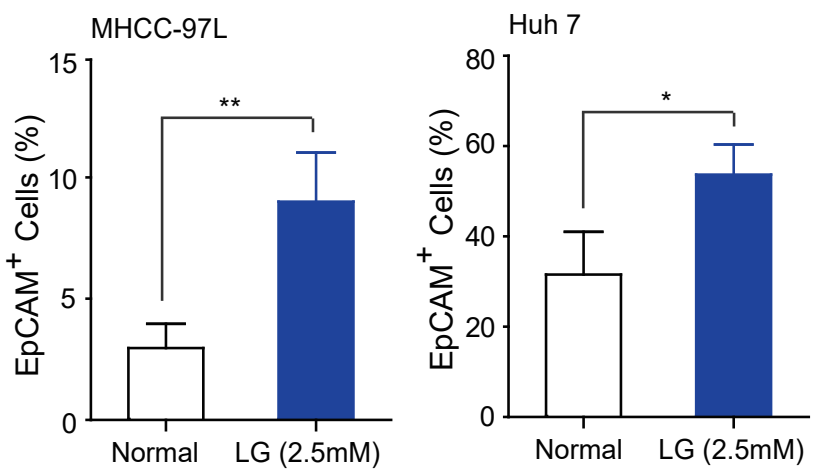

C
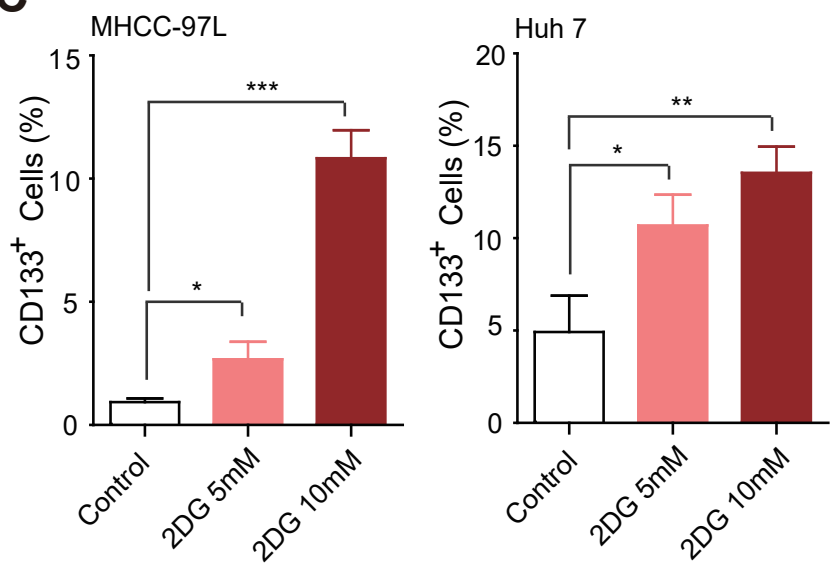

Primary HCC cells

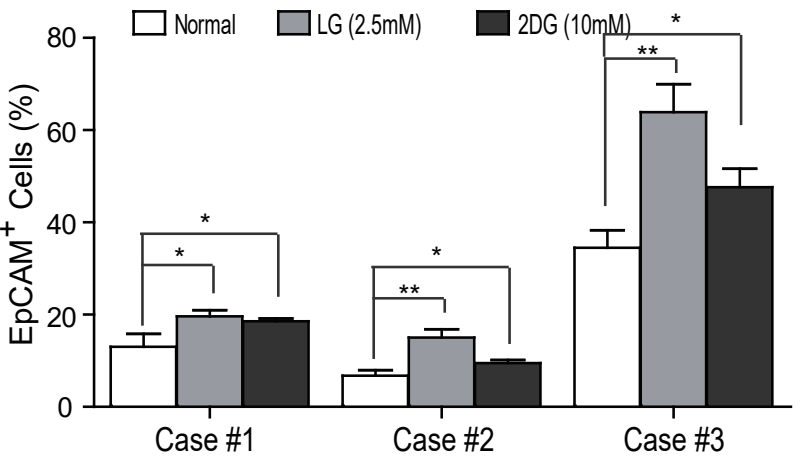


A

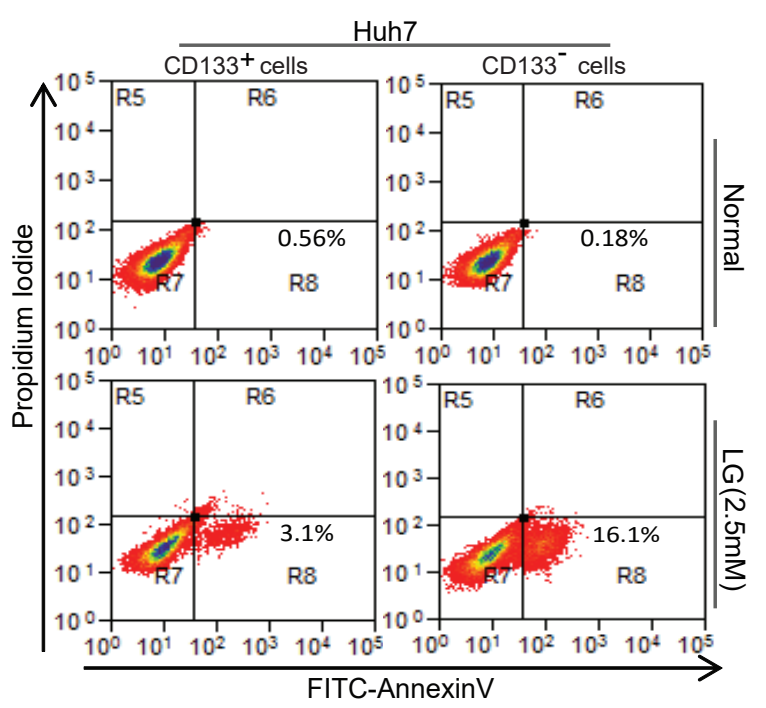

D

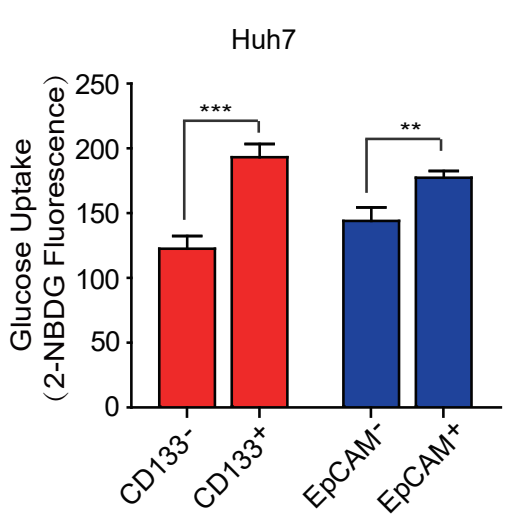

B
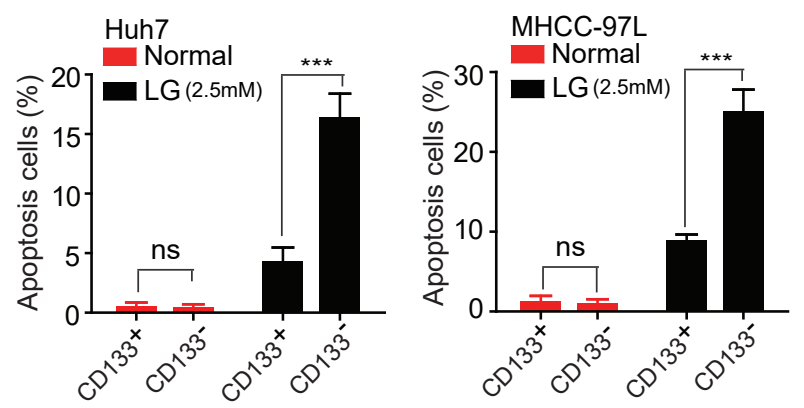

C

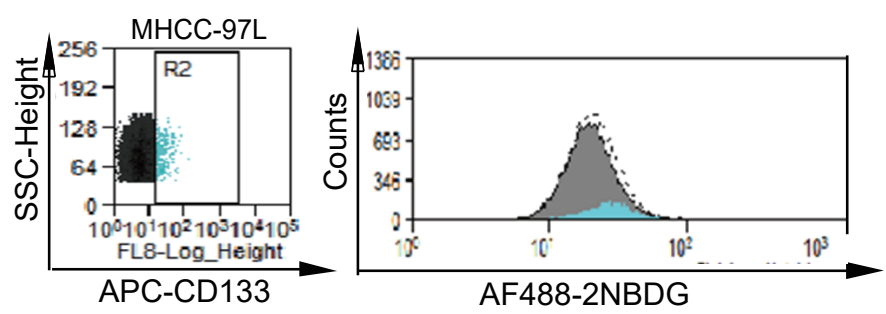

E
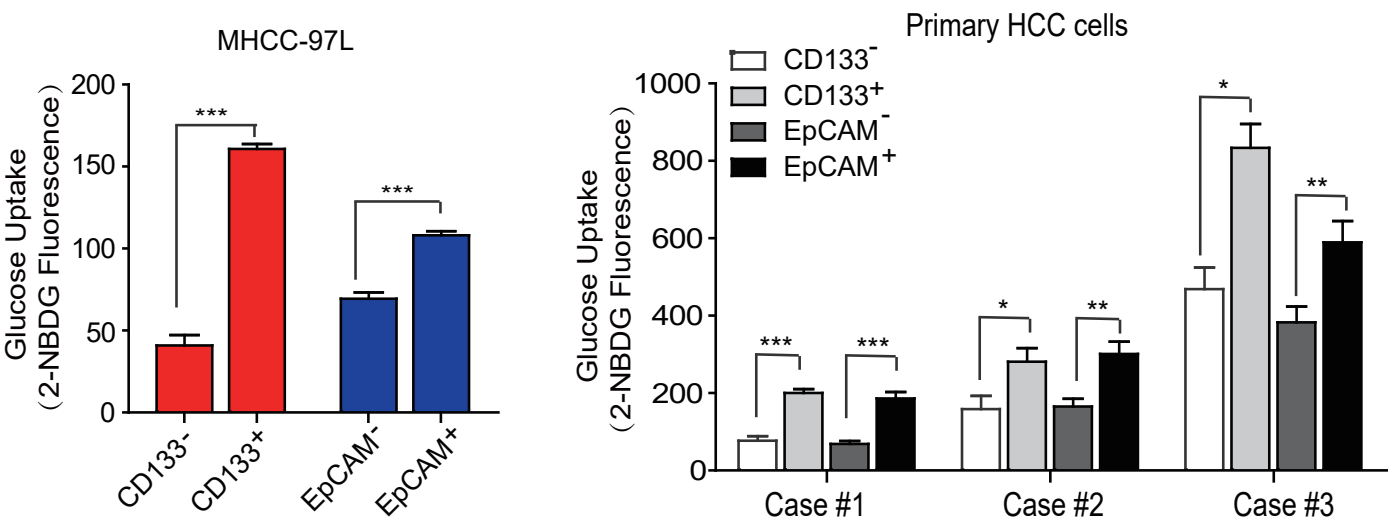
Figure 3

A

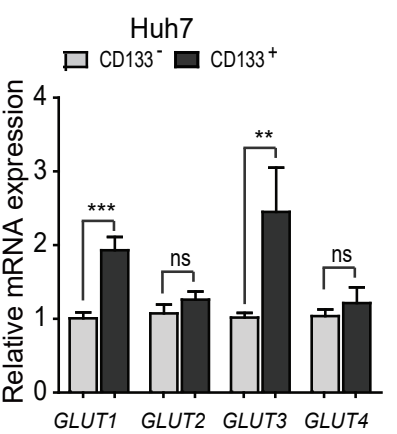

D

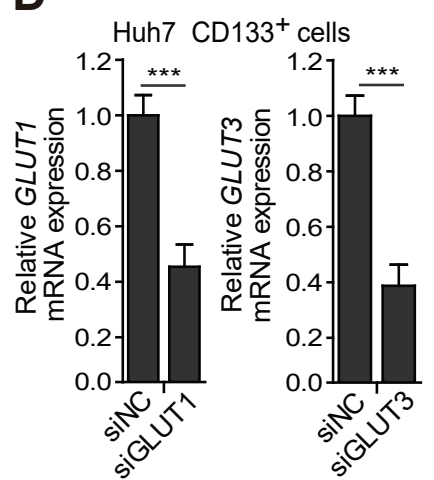

MHCC-97L

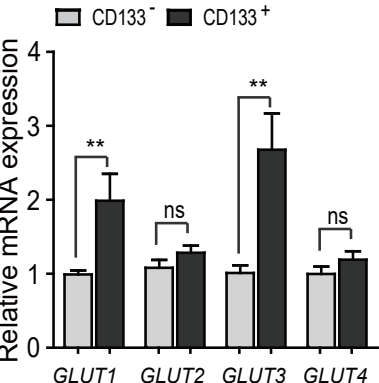

E

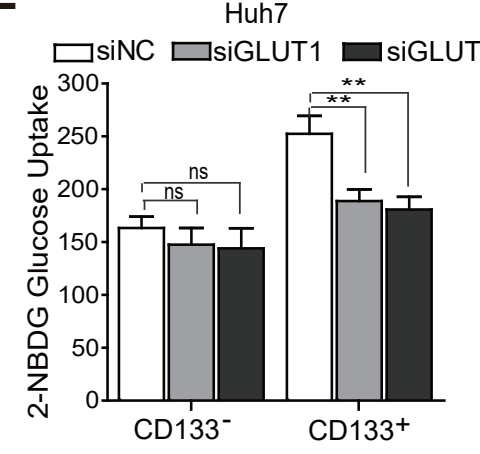

B Primary HCC cells

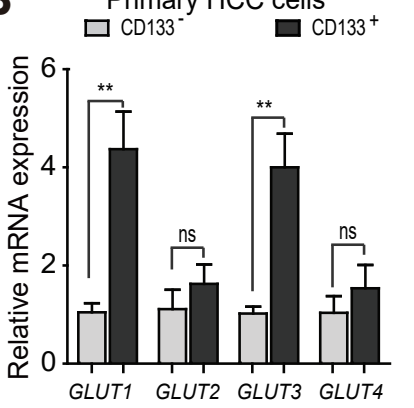

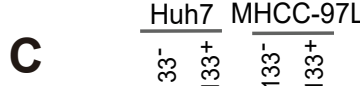
㝴

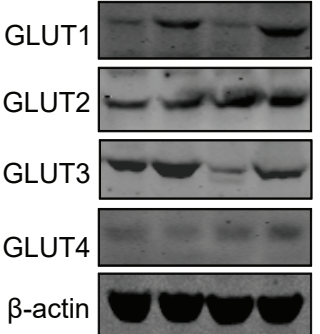

Primary HCC cells m $\stackrel{m}{m} \stackrel{m}{m} \stackrel{m}{\infty}$ 守

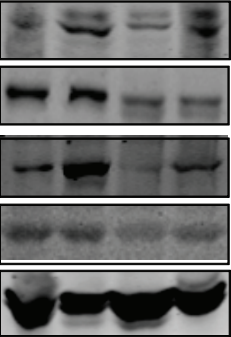

Patient 1 Patient 2
F

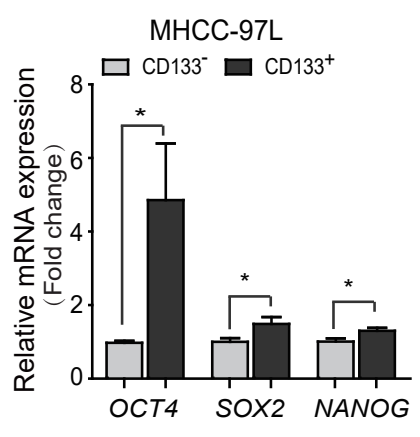

Huh7

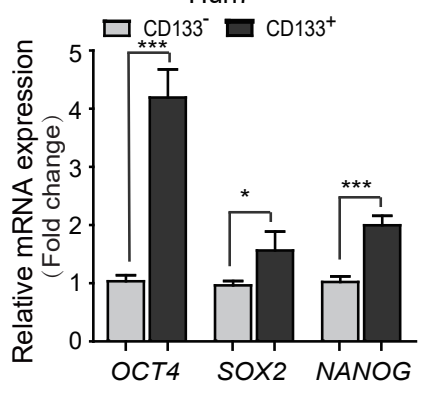

G Primary HCC Cells (87 cases)

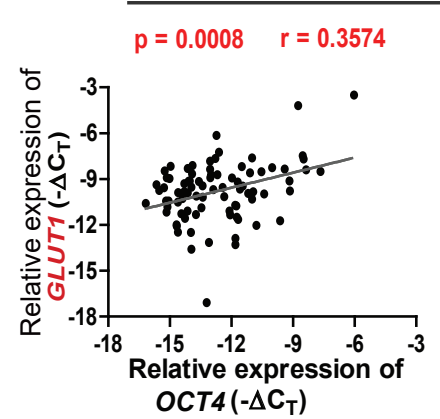

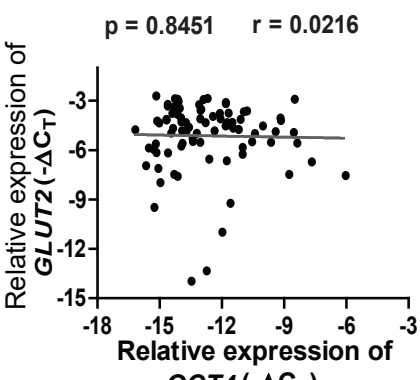

$\operatorname{OCT4}\left(-\Delta \mathrm{C}_{\mathrm{T}}\right)$

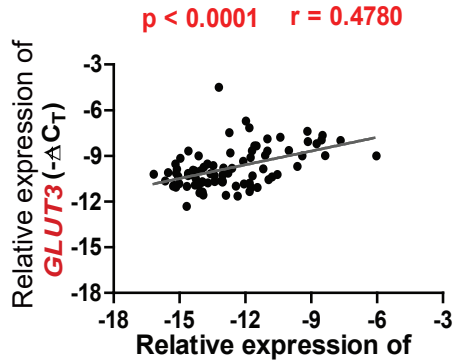

OCT4 $\left(-\Delta \mathrm{C}_{\mathrm{T}}\right)$

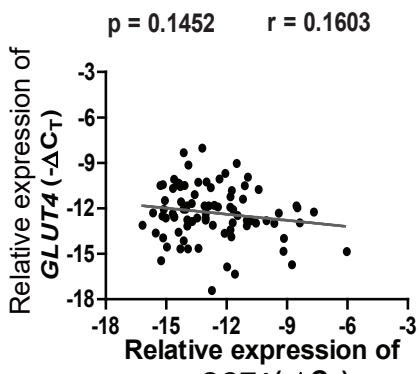

OCT4 $\left(-\Delta \mathrm{C}_{\mathrm{T}}\right)$ 


\section{Figure 4}

A

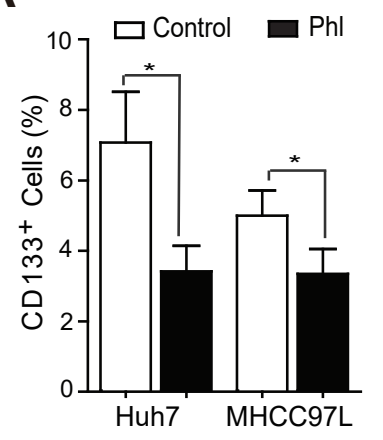

D

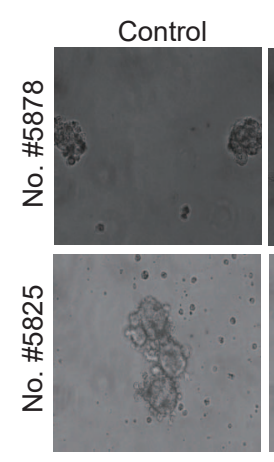

$\mathbf{F}$

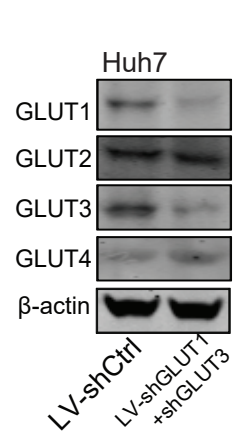

G
B

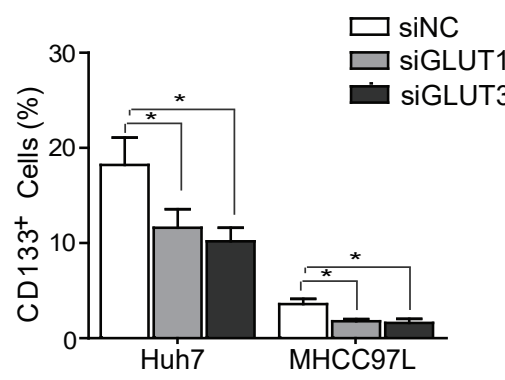

C

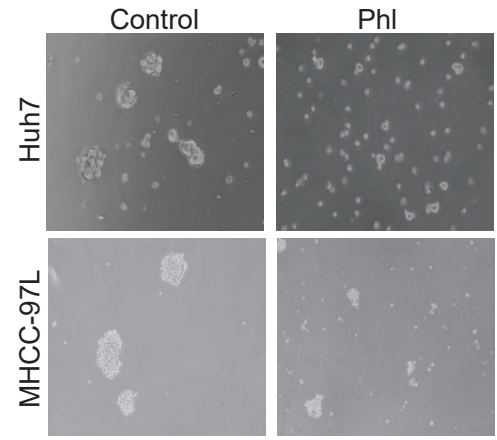

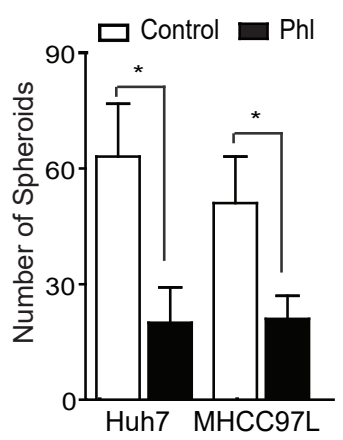

E
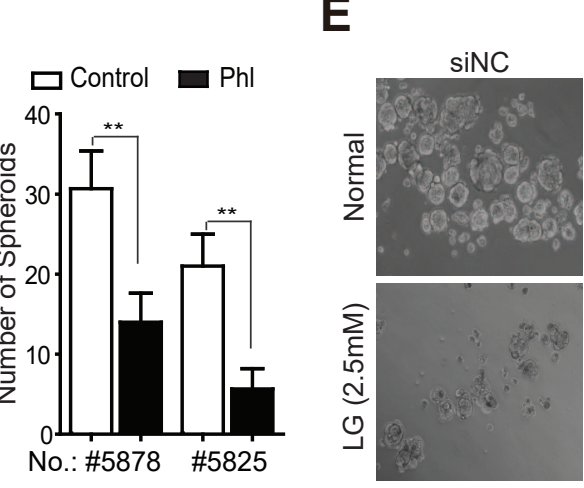

Huh7 CD $133^{+}$Cells
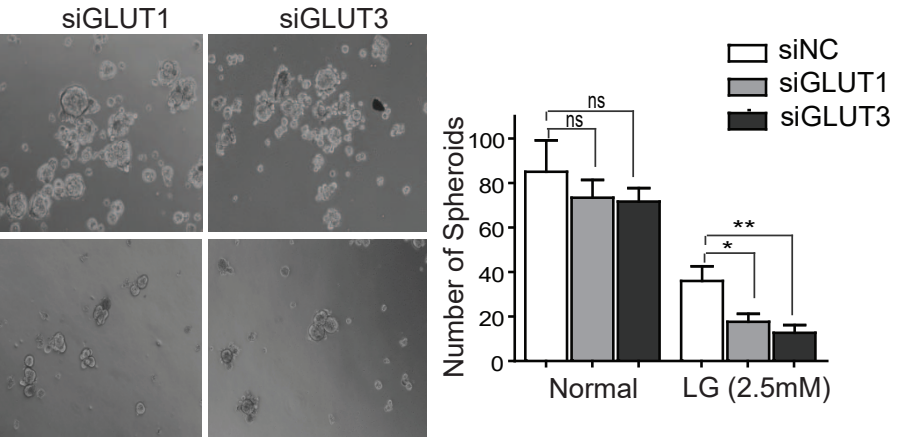

H

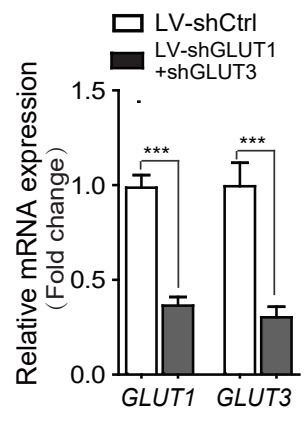

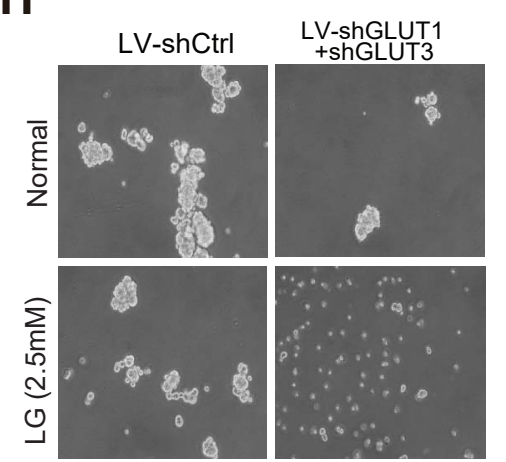

Huh7 CD $133^{+}$Cells

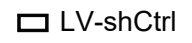

- $\begin{gathered}\text { LV-shGLUT } \\ \text { +shGLUT3 }\end{gathered}$

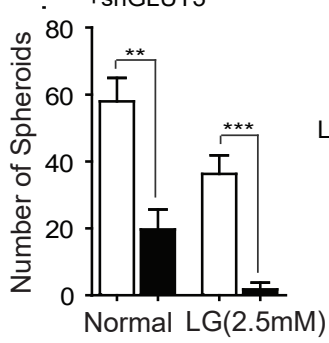

Huh7 CD133+ Cells Xenograft Model

LV-shCtrl

V-shGLUT1 +shGLUT3

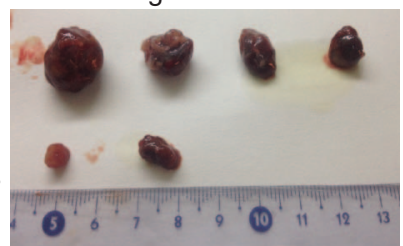


Figure 5

A Huh7

$\square$ Normal CD133+

$\square$ LG (2.5mM) CD133+

Normal CD133

$\square$ LG (2.5mM) CD133-

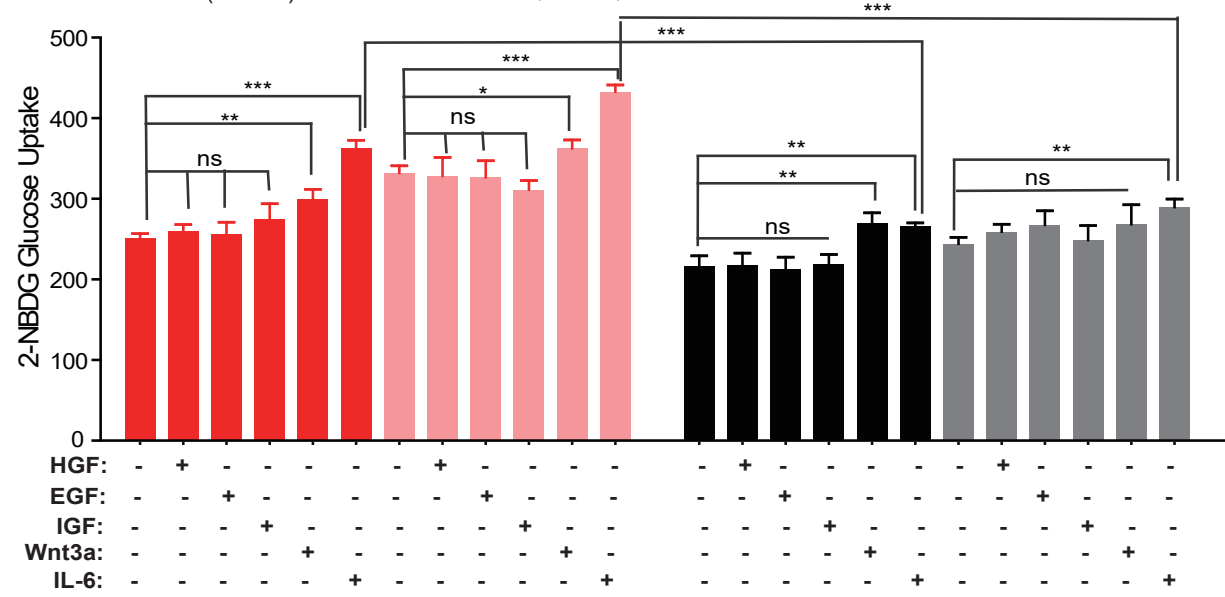

C

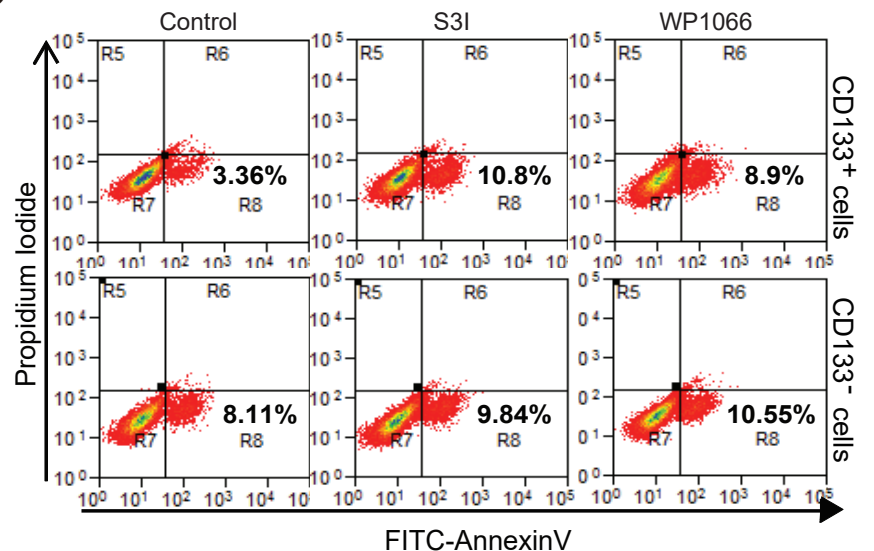

Huh7 LG (2.5mM)

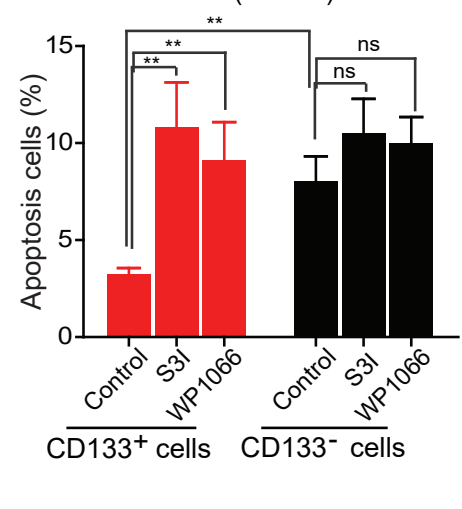

B

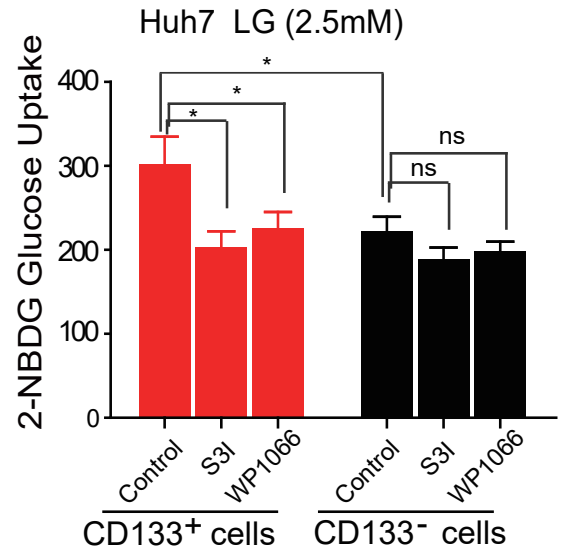

D

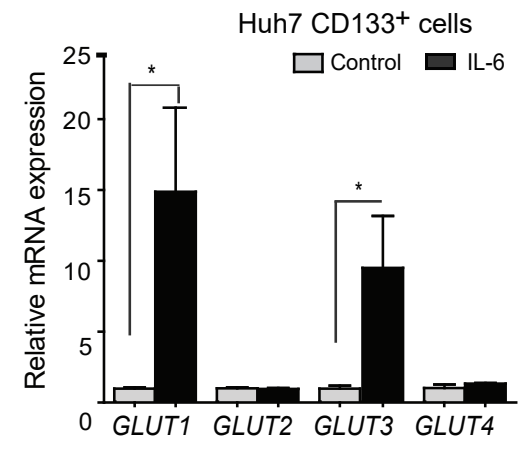




\section{Figure 6}

A

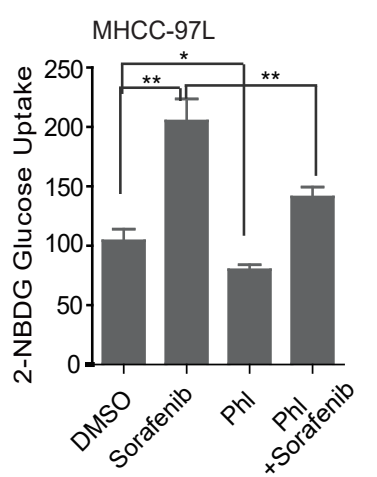

C
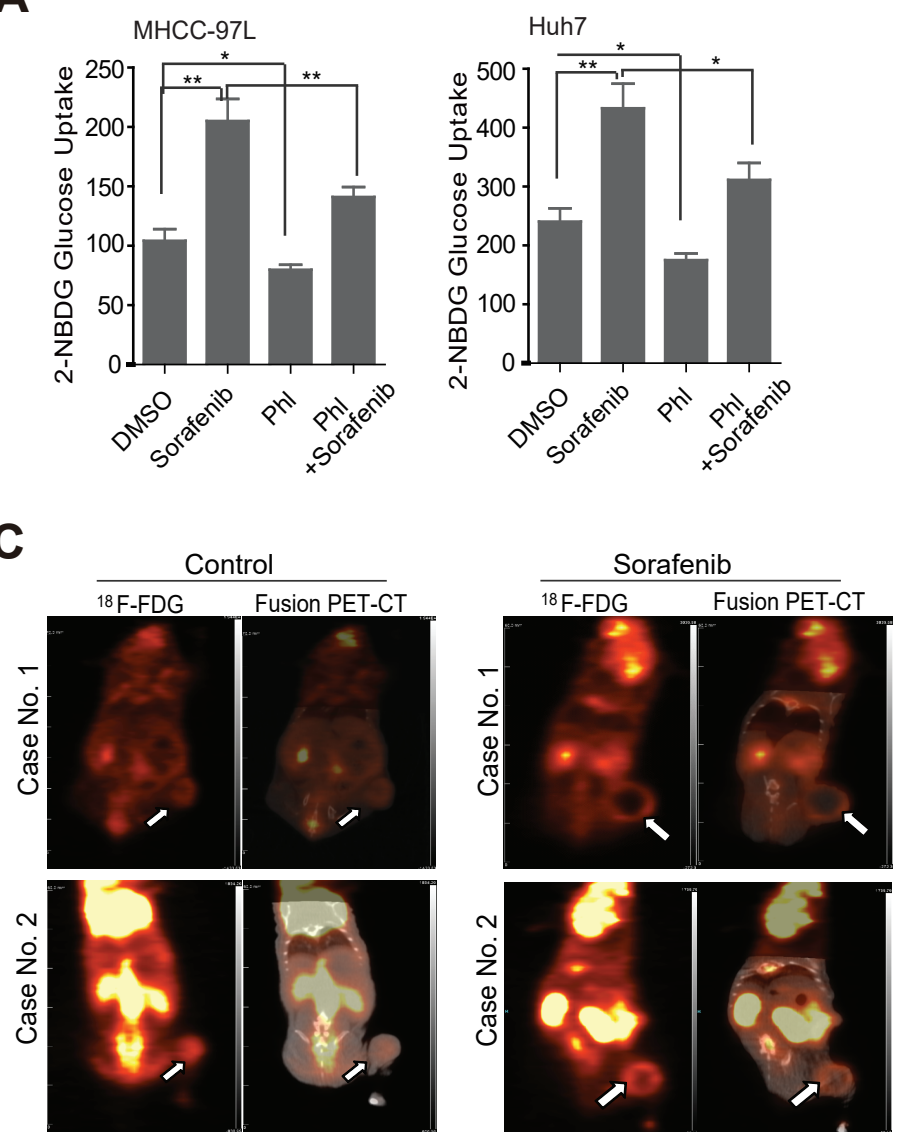

$\mathbf{F}$

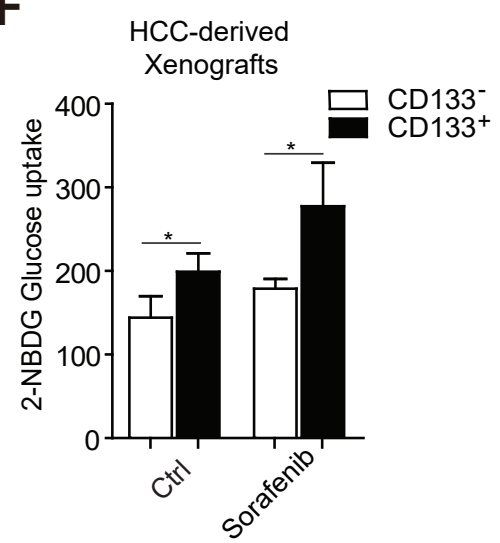

B
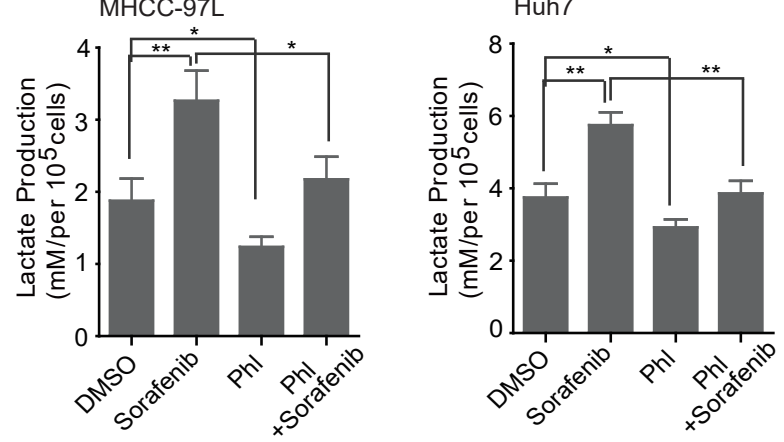

D

HCC-derived
Xenografts

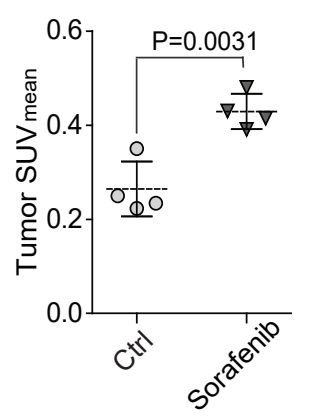

E HCC-derived Xenografts

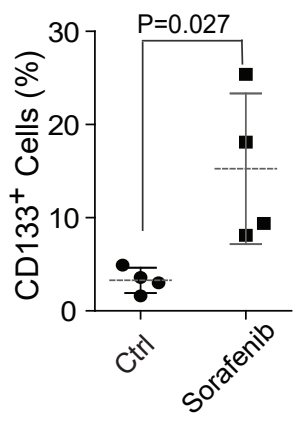

H
G

Huh7 CD133+ cells Control Sorafenib Case No.: \#1 \#2 \#3 \#1 \#2 \#3 GLUT1 GLUT2 GLUT3 GLUT4 $\beta$-actin

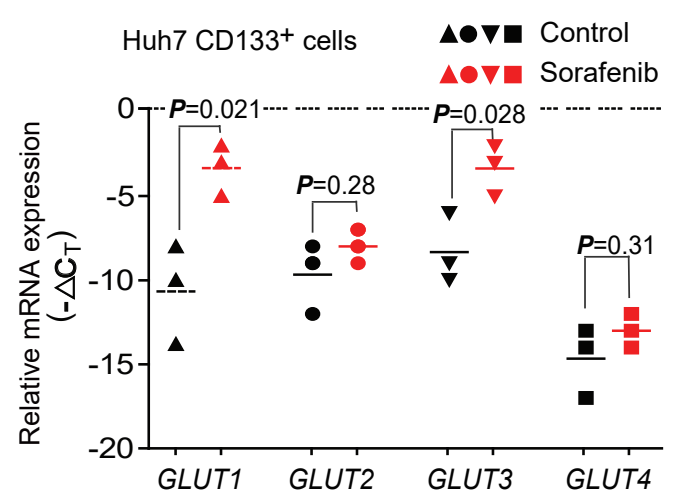




\section{Figure 7}

A
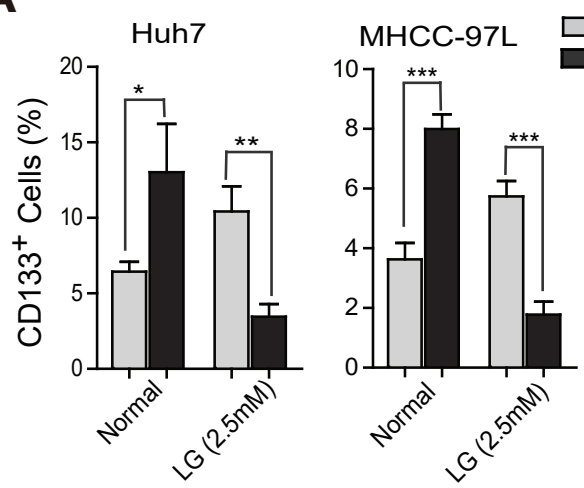

B

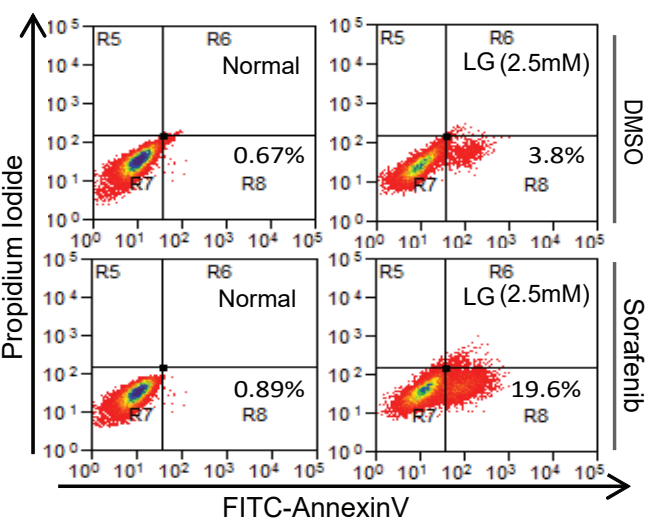

Huh 7 CD $133^{+}$Cells

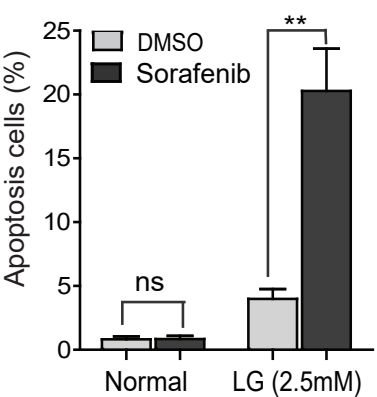

C

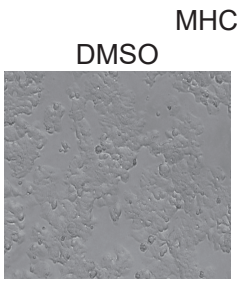

Phl

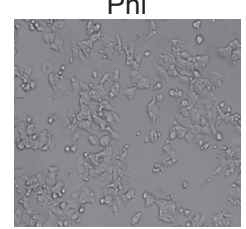

7L

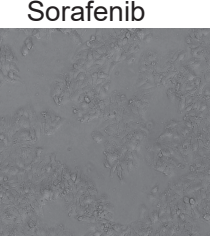

Phl+Sorafenib

D
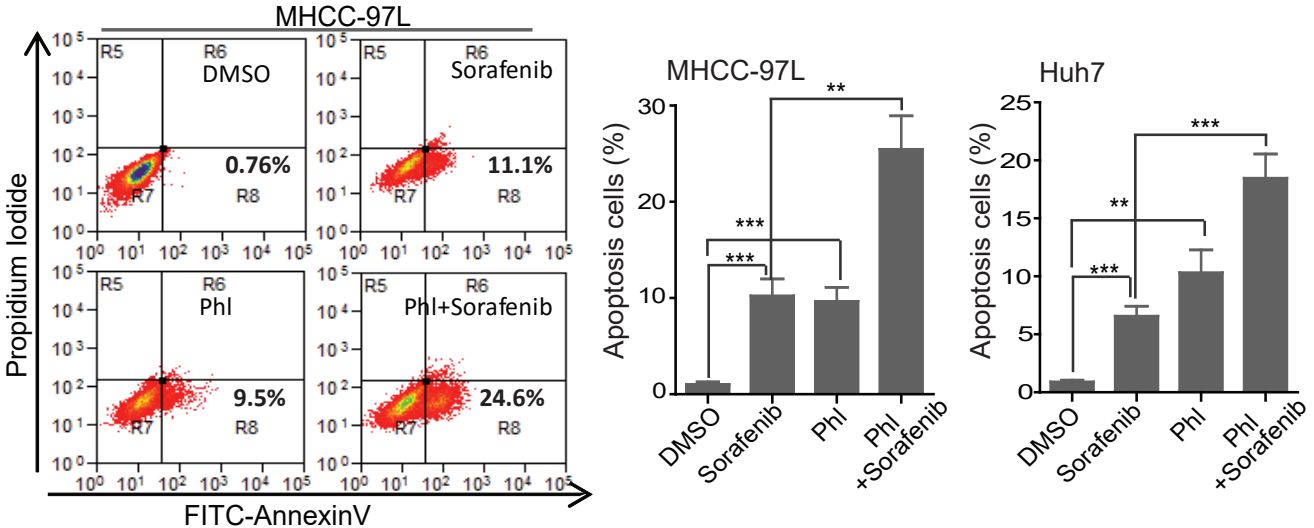

E

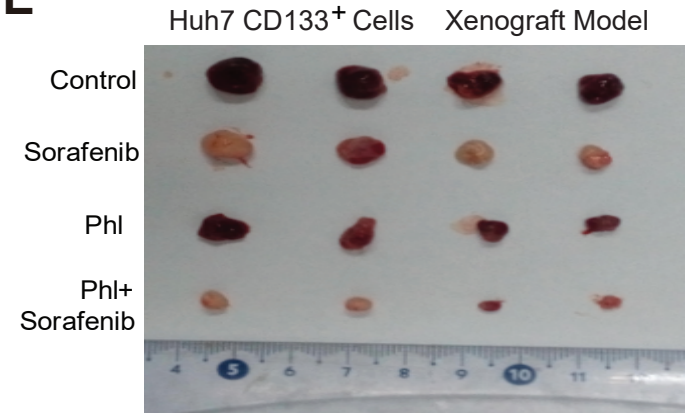

F

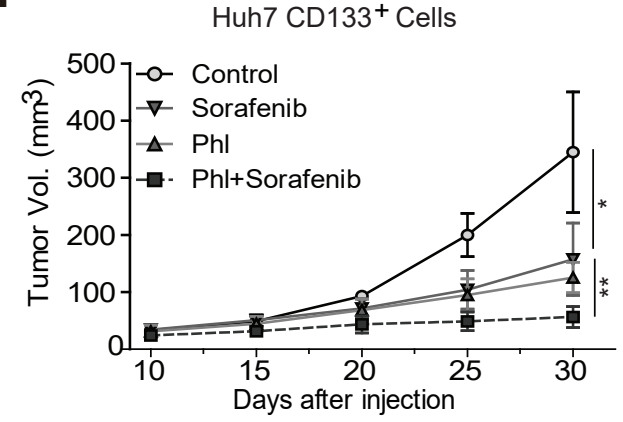

